Encyclopedia of Social Network Analysis and Mining 
Reda Alhajj - Jon Rokne Editors

\section{Encyclopedia of Social Network Analysis and Mining}

Second Edition

With 847 Figures and 272 Tables

第 Springer 


\section{Editors}

Reda Alhajj

Department of Computer Science

University of Calgary

Calgary, AB, Canada

\author{
Jon Rokne \\ Department of Computer Science \\ University of Calgary \\ Calgary, AB, Canada
}

ISBN 978-1-4939-7130-5

ISBN 978-1-4939-7131-2 (eBook)

ISBN 978-1-4939-7132-9 (print and electronic bundle)

https://doi.org/10.1007/978-1-4939-7131-2

Library of Congress Control Number: 2018934718

1st edition: (C) Springer Science+Business Media New York 2014

(C) Springer Science+Business Media LLC, part of Springer Nature 2018

This work is subject to copyright. All rights are reserved by the Publisher, whether the whole or part of the material is concerned, specifically the rights of translation, reprinting, reuse of illustrations, recitation, broadcasting, reproduction on microfilms or in any other physical way, and transmission or information storage and retrieval, electronic adaptation, computer software, or by similar or dissimilar methodology now known or hereafter developed.

The use of general descriptive names, registered names, trademarks, service marks, etc. in this publication does not imply, even in the absence of a specific statement, that such names are exempt from the relevant protective laws and regulations and therefore free for general use.

The publisher, the authors and the editors are safe to assume that the advice and information in this book are believed to be true and accurate at the date of publication. Neither the publisher nor the authors or the editors give a warranty, express or implied, with respect to the material contained herein or for any errors or omissions that may have been made. The publisher remains neutral with regard to jurisdictional claims in published maps and institutional affiliations.

Printed on acid-free paper

This Springer imprint is published by the registered company Springer Science+Business Media LLC part of Springer Nature.

The registered company address is: 233 Spring Street, New York, NY 10013, U.S.A. 
To the memory of my parents and to my wife Dima Halabi; my daughters Aya, Loubaba, and Lama; and my sons Suleiman and Mohammed Taha.

Reda Alhajj

To Angela, Knut, and Olav for support with this major venture.

Jon Rokne 


\section{Preface to Second Edition}

The second edition of the Encyclopedia of Social Network Analysis and Mining (ESNAM) is an updated version of the very successful first edition which appeared in 2014. The new version has new sections and new contributed entries as well as updated versions of entries from the first edition. The aim is to keep the encyclopedia current by covering the state of the art in social network analysis and mining and providing an up-to-date online and print source that encompasses a comprehensive collection of entries in the areas of social networks and the mining of social networks.

While social networks initially emerged as an area of sociology, and mining (i.e., data mining) is an area of informatics, the application of mining to online social networks is considered to be a new and important interdisciplinary area of research. ESNAM surveys this new area while also including basic supporting information from both social networks (including the fundamentals of network analysis) and mining as well as introductions to fundamental analytical tools relevant to the area.

This new area is essentially an outcome of the widespread introduction of electronic communication. One can hardly imagine Facebook, Twitter, and other social networking sites having the current attraction for large segments of population without the communication convenience provided by the Internet. The ability to connect humans over great distances has therefore transformed the notion of social networks from the previously studied relatively small and geographically limited social networks to those spanning the globe (and possibly further into space in the future). Together with this ability to seamlessly communicate over arbitrary distances, an ability to collect data about this communication has been enabled. Data on who communicates with whom can be gathered and refined into a social network setting. Once the social connections have been established, they can be analyzed and potentially interesting observations can be extracted.

Social networks have taken on new and important functions. For example, in politics they can be used to influence political views. An important example of this is the Twitter network where important individuals influence opinions by having many followers who retweet their utterances. Countries can subordinate social networks for propaganda purposes, especially to influence opinions in other possible hostile countries.

Social networks are ubiquitous. They can be found in many areas of science and social science. They can be classified in various ways. Explicit social 
networks model directly known relationships between actors. This is in contrast to implicit social networks where the links between actors can only be inferred by deeper analysis of the available data. Facebook, Twitter, and MySpace are good examples where explicit social networks can be found. Implicit social networks can be found by analyzing Amazon and Netflix logs of activities, for example, for common interests such as political viewpoints or hobbies.

For another classification, stationary items such as pieces of code, stock items, or routers in a network can be viewed as network actors and be grouped together into social communities. Similarly, dynamic actors, such as animals, humans, insects, genes, proteins, etc., can form social communities.

Understanding social communities of dynamic and moving objects like stars or humans, which may be bound by various physical or psychosocial forces, is fairly intuitive, and their analysis can provide interesting knowledge that is most often easy to understand. Social communities of abstract items tend to be less intuitive, but the analysis of these communities can also result in interesting discoveries such as finding groups of tables in databases that are related and mostly accessed together. These discoveries and insights can lead to better plans or to improved algorithms for prefetching data.

Graphical representations are used to depict the interactions between individuals in a social network and are essential for understanding how information items, innovations, and disease occurrences are connected. Edges in the graph may represent social interactions, organizational structures, physical proximity, or even more abstract interactions such as hyperlinks or similar. Graph theoretical principles have generally influenced the overall terminology used in social networks and many of the techniques used to analyze the networks as well.

The fundamental issues in social network research were broadly defined in the 1930s within the field of sociology, though some works conducted in the 19th century can be witnessed as described in the literature. The early research focused on human social communities and interactions. Rapid progress was made in the 1970s when researchers broadened the study of social networks from a sociological perspective of a study of communities of humans to other animals and to more general communities. For the symbiosis with biology, the term "sociobiology" was introduced in E. O. Wilson's Sociobiology: The New Synthesis.

More recently, the interest in studying human social networks has exploded with the appearance of global web-based communities. People from all over the world are directly or indirectly connected by popular social networks such as Facebook and Twitter rather than by physical proximity as they were in the past. These networks have turned into powerful media for exchanging views and influencing others. The sizes of these networks are growing fast, and traditional techniques for network analysis must yield automated methods for analyzing and handling them.

The study of social communities is extremely multidisciplinary, requiring expertise from computer science, sociology, behavioral science, mathematics, statistics, etc. Data mining techniques and analyses have been applied to gain valuable discoveries with pervasive social and economic impacts in many 
areas of applied social network research. From the social perspective, application areas include antiterrorism strategies, crime data analysis, disease biomarkers, common hobbies, family relationships, social functions, occupations, and friendship, among others. From the economic perspective, the analyses may lead to certain target customer groups, the development of drugs, etc.

The Encyclopedia of Social Network Analysis and Mining is the first major reference work to integrate all the fundamental concepts and research directions within the areas of social networks and the application of data mining to social networks in a single set of comprehensive and authoritative volumes:

- It presents the first comprehensive coverage of all fields, including computer science, statistics, social and cultural anthropology, and informatics in general, that contribute to an understanding of the structure and function of social networks.

- It is designed to appeal to an interdisciplinary audience with the level of expertise ranging from undergraduates to professionals.

- It explains the science and technology behind the fast-growing global social network avenues for exchanging views and influencing others.

- It describes social network applications to knowledge mining and discovery, ranging from web-mining algorithms to health informatics and epidemiology to antiterrorism strategies.

- It addresses privacy, security, ethical, and civil liberty issues for social networks. 


\section{Preface to First Edition}

The Encyclopedia of Social Network Analysis and Mining (ESNAM) is the first online and print reference work that encompasses a comprehensive collection of articles in the areas of social networks and the mining of social networks.

While social networks is an area of sociology, and mining (i.e., data mining) an area of informatics, the application of mining to online social networks is considered to be a new and important interdisciplinary area of research. ESNAM surveys this new area while also including basic supporting information from both social networks (including the fundamentals of network analysis) and mining.

This new area is essentially an outcome of the widespread introduction of electronic communication. One can hardly imagine Facebook, Twitter, and online pornography existing without the convenience of a widespread Internet. The ability to connect humans over great distances has therefore transformed the notion of social networks from the previously studied relatively small and geographically limited social networks to social networks spanning the globe (and possibly further into space in the future). Together with this ability to seamlessly communicate over arbitrary distances, an ability to collect data about this communication has been enabled. Data on who communicates with whom can be gathered and refined into a social network setting. Once the social connections have been established, they can be analyzed and potentially interesting observations can be extracted.

Social networks are ubiquitous. They can be found in many areas of science and social science. They can be classified in various ways. Explicit social networks model directly known relationships between actors. This is in contrast to implicit social networks where the links between actors can only be inferred by deeper analysis of the available data. Facebook, Twitter, and MySpace are good examples where explicit social networks can be found. Implicit social networks can be found by analyzing Amazon and Netflix logs of activities, for example, for common interests such as political viewpoints or hobbies.

For another classification, stationary items such as pieces of code, stock items, or routers in a network can be viewed as network actors and be 
grouped together into social communities. Similarly, dynamic actors, such as animals, humans, insects, genes, proteins, etc., can form social communities.

Understanding social communities of dynamic and moving objects like stars or humans, which may be bound by various physical or psychosocial forces, is fairly intuitive, and their analysis can provide interesting knowledge that is most often easy to understand. Social communities of abstract items tend to be less intuitive, but the analysis of these communities can also result in interesting discoveries such as finding groups of tables in databases that are related and mostly accessed together. These discoveries and insights can lead to better plans or to improved algorithms for prefetching data.

Graphical representations are used to depict the interactions between individuals in a social network and are essential for understanding how information items, innovations, and disease occurrences are connected. Edges in the graph may represent social interactions, organizational structures, physical proximity, or even more abstract interactions such as hyperlinks or similar. Graph theoretical principles have generally influenced the overall terminology used in social networks and many of the techniques used to analyze the networks as well.

The fundamental issues in social network research were broadly defined in the 1930s within the field of sociology. The early research focused on human social communities and interactions. Rapid progress was made in the 1970s when researchers broadened the study of social networks from a sociological perspective of a study of communities of humans to other animals and to more general communities. For the symbiosis with biology, the term "sociobiology" was introduced in E. O. Wilson's "Sociobiology: The New Synthesis."

More recently, the interest in studying human social networks has exploded with the appearance of global web-based communities. People from all over the world are directly or indirectly connected by popular social networks such as Facebook and Twitter rather than by physical proximity as they were in the past. These networks have turned into powerful media for exchanging views and influencing others. The sizes of these networks are growing fast, and traditional techniques for network analysis must yield automated methods for analyzing and handling them.

The study of social communities is extremely multidisciplinary, requiring expertise from computer science, sociology, behavioral science, mathematics, statistics, etc. Data mining techniques and analyses have been applied to gain valuable discoveries with pervasive social and economic impacts in many areas of applied social network research. From the social perspective, application areas include antiterrorism strategies, crime data analysis, disease biomarkers, common hobbies, family relationships, social functions, occupations, and friendship. From the economic perspective, the analyses may lead to certain target customer groups, the development of drugs, etc.

The Encyclopedia of Social Network Analysis and Mining is the first major reference work to integrate all the fundamental concepts and research 
directions within the areas of social networks and the application of data mining to social networks in a single set of comprehensive and authoritative volumes:

- It presents the first comprehensive coverage of all fields, including computer science, statistics, social and cultural anthropology, and informatics in general, that contribute to an understanding of the structure and function of social networks.

- It is designed to appeal to an interdisciplinary audience and level of expertise ranging from undergraduates to professionals.

- It explains the science and technology behind the fast-growing global social network avenues for exchanging views and influencing others.

- It describes social network applications to knowledge mining and discovery ranging from web mining algorithms to health informatics and epidemiology to antiterrorism strategies.

- It addresses privacy, security, ethical, and civil liberty issues for social networks. 


\section{Acknowledgments}

The new edition of this encyclopedia could not have been successfully completed without the support and contribution of the Springer staff, advisory board members, section editors, authors, and reviewers; we deeply thank them all.

A special thank-you to Esther, Barbara, Chris, and Lydia from Springer. They have worked hard and closely with us at all stages of this project. We are very grateful for the excellent work and dedication they have contributed to make this a successful project. 


\section{List of Topics}

Applied Computational Geometry and Graph Theory for Social Networks

Section Editor: Richard Chbeir and

Mick J. Ridley

Community Detection in Social Network: An

Experience with Directed Graphs

Creating a Space for Collective Problem-Solving

Evolving Social Graph Clustering

Sociograph Representations, Concepts, Data, and Analysis

\section{Basics of Social Network Model Construction and Evolution}

\section{Section Editor: Ralf Klamma}

Modeling Social Preferences Based on Social Interactions

Network Representations of Complex Data

\section{Crowd Sourcing, Opinion Mining, Fusion and Influence Analysis}

Section Editor: Guandong $X u$ and Peng Cui

Behavior Analysis in Social Networks

Behavior Modeling in Social Networks

Cross-Platform Social Network Analysis

Crowdsourced Social Data for Recommending

Tourist Itineraries

Detecting Crowdturfing in Social Media

Identifying Spam in Reviews

Influence Maximization Model
Mathematical Model for Propagation of Influence in a Social Network

Sentiment Analysis in Social Media, Aspect

Extraction for

Spam Detection on Social Networks

\section{Current and Future Research Directions}

Section Editor: Andrea Tagarelli and Fabrizio Silvestri

Aesthetics in Social Media

Analysis and Planning of Urban Networks

Anonymization and De-anonymization of Social

Network Data

Arts and Humanities, Complex Network

Analysis of

Benchmarking for Graph Clustering and Partitioning

Classical Algorithms for Social Network Analysis: Future and Current Trends

Community Detection: Current and Future Research Trends

Economic Network Analysis Based on Infection Models

Link Prediction: A Primer

Mining Trends in the Blogosphere

Multiple Social Networks, Data Models and

Measures for

Multiplex Networks

Network Actors Within Entrepreneurial

Networks: The Current State of Research

Network Science Research Dynamic

Paths in Complex Networks 
Privacy in Social Networks, Current and Future Research Trends on

Recommender Systems Using Social Network Analysis: Challenges and Future Trends

Sampling Effects in Social Network Analysis

Social Networks and Politics

Social-Spatiotemporal Analysis of Topical and

Polarized Communities in Online Social

Networks

Spatial Networks

Statistical Research in Networks: Looking

Forward

Temporal Networks

Nature of Social Structures

Visualization of Large Networks

Data Collection, Data Extraction, and Data Preparation for Social Networks Analysis and Mining

Section Editor: V.S. Subrahmanian and Jeffrey Chan

Collecting Qualitative Data to Enhance Social

Network Analysis and Data Mining

Desire Lines in Big Data

Imputation of Missing Network Data

Incentives in Collaborative Applications

Network Data Collected via Web

Outlier Detection with Uncertain Data Using

Graphics Processors

Quality of Social Network Data

Query Answering in the Semantic Social Web:

An Argumentation-Based Approach

Questionnaires for Measuring Social Network Contacts

Scaling Subgraph Matching Queries in Huge

Networks

Socialbots

Data Management for Social Networks and Social Media

Section Editor: Jaideep Srivastava and Abdullah Uz Tansel

Querying Volatile and Dynamic Networks

Social Provenance
Social Search and Querying

Stream Querying and Reasoning on Social Data

Transforming Social Networks Data

\section{Data Mining and Machine Learning \\ Techniques for Social Networks}

\section{Section Editor: Fakhri Karray}

Automatic Document Topic Identification Using Social Knowledge Network

Collective Classification

Collective Classification: Structural Features

Game-Theoretic Framework for Community Detection

Microtext Processing

Multi-classifier System for Sentiment Analysis and Opinion Mining

Network Anomaly Detection Using Co-clustering Real-Time Detection of Topics in Twitter Streams Recommender Systems: Models and Techniques Relative Validity Criteria for Community Mining Algorithms

Subgraph Extraction for Trust Inference in Social Networks

Topic Modeling in Online Social Media, User

Features and Social Networks for

User Behavior in Online Social Networks:

Influencing Factors

History of Social Networks: Past, Present and future

\section{Section Editor: Talel Abdessalem and Rokia}

Missaoui

Data Mining Techniques for Social Networks Analysis

Deviant and Criminal Uses of Social Networks

Disaster Response and Relief, VGI Volunteer Motivation in

E-government

E-commerce and Internet Business

East Asian Social Networks

Election Forecasting: Scientific Approaches

Futures of Social Networks: Where Are Trends Heading?

Gaming and Virtual Worlds 
History and Evolution of Social Network Visualization

History of Networks at Columbia University

Learning Networks

Mobile Communication Networks

Network Analysis in French Sociology and Anthropology

Network Management and Governance

Networks at Harvard University Sociology

Networks in Geography

Networks in Rural Sociology

Networks in Social Psychology: Beginning with Kurt Lewin

Networks in the Twenty-First Century

Online Communities

Online Healthcare Management

Origins of Social Network Analysis

Personal Networks: Ties, Space, and the Internet

Policy Networks: History

Political Networks

Pornography Online

Semantic Social Networks

Social Bookmarking or Tagging

Social Capital

Social History of Computing and Online Social Communities

Social Media and Social Networking in Political Campaigns/Movements

Social Media, Definition, and History

Social Network Analysis in an Age of Digital Information

Social Networking on the World Wide Web

Wikipedia Collaborative Networks

Internet, Web 2.0, Web Services and Semantic Web

\section{Section Editor: Steffen Staab, Thomas Gottron and Stefan Schlobach}

Automated Reasoning

Cloud Computing

Collective Intelligence: Overview

Crowdsourcing and Human Computation:

Introduction

Description Logics

Folksonomies

HTML
Linked Open Data

Ontology Matching

RDF

RIF: The Rule Interchange Format

Recommender Systems, Semantic-Based

Retrieval Models

SPARQL

Sentiment Analysis in Social Media

Server-Side Scripting Languages

Service Discovery

Social Network Datasets

Spectral Evolution of Social Networks

Tag Clouds

Unicode

WSDL

Web Archives

Web Mash-Ups

Web Ontology Language (OWL)

Web Science

Web Service Composition

Web Service Infrastructure Patterns

Weblog Analysis

XPath/XQuery

XSLT

e-Science

\section{Linear Algebra and Statistics for Social Networks}

\section{Section Editor: Suheil Khoury}

Actor-Based Models for Longitudinal Networks Barycentric Discriminant Analysis

Canonical Correlation Analysis

Clustering Algorithms

Correspondence Analysis

Distance and Similarity Measures

Effect Size as a Measure of Difference Between Two Populations

Eigenvalues: Singular Value Decomposition

Gibbs Sampling

Independent Component Analysis

Iterative Methods for Eigenvalues/Eigenvectors

Least Squares

Markov Chain Monte Carlo Model

Matrix Algebra, Basics of

Matrix Decomposition

Principal Component Analysis 
Probabilistic Analysis

Probabilistic Graphical Models

Probabilistic Logic and Relational Models

Probability Matrices

Queueing Theory

Ranking Methods for Networks

Regression Analysis

Semi-Discrete Decomposition

Semirings and Matrix Analysis of Networks

Simulated Datasets

Simulations

Spatial Statistics

Spectral Analysis

Synthetic Data Sets

Theory of Probability: Basics and Fundamentals

Theory of Statistics: Basics and Fundamentals

Univariate Descriptive Statistics

\section{Online Social Networks, Social Networking Sites and Social Media}

\section{Section Editor: Irwin King and Jie Tang}

Analysis and Mining of Tags, (Micro)Blogs, and Virtual Communities

Collective Intelligence for Crowdsourcing and Community Q\&A

Game Theory and Social Networks

Graph Classification in Heterogeneous Networks Inferring Social Ties

Location-Based Social Network Analysis

Matrix Factorization Framework for Overlapping Community Detection

Models for Community Dynamics

Social Influence Analysis

Social Recommendation in Dynamic Networks

Topology of Online Social Networks

User Sentiment and Opinion Analysis

Privacy and Security Issues in Social Networks

\section{Section Editor: Henric Johnson and Felix Wu}

Consequences of Publishing Real Personal Information in Online Social Networks Crime Prevention, Dataveillance, and the Regulation of Information Communication Technologies
Ethical Issues Surrounding Data Collection in Online Social Networks

Ethics of Social Networks and Mining

Facebook's Challenge to the Collection Limitation Principle

Graphical User Interfaces for Privacy Settings

Legal Implications of Social Networks

Online Identities and Social Networks in

Organizational Settings

Online Privacy Paradox and Social Networks

Online Social Network Phishing Attack

Online Social Network Privacy Management

Privacy Issues for SNS and Mobile SNS

Privacy Preservation and Location-Based Online Social Networking

Privacy and Disclosure in a Social Networking Community

Reconnaissance and Social Engineering Risks as Effects of Social Networking

Self-Efficacy Versus Expertise

Social Media Policy in the Workplace: User Awareness

\section{Sentiment Analysis and Recommender Systems}

\section{Section Editor: Giovanni Semeraro and Cataldo Musto}

Community Detection and Recommender Systems

Emotions and Personality in Recommender Systems

Recommender Systems Based on Linked Open Data

Recommender Systems Based on Social Networks

Recommender Systems Evaluation

Recommender Systems, Basics of

Relational Network Classification and Its Applications in Recommender Systems Semantic Sentiment Analysis of Twitter Data Sentiment Analysis of Microblogging Data Sentiment Analysis of Reviews Sentiment Analysis, Basic Tasks of Sentiment Quantification of User-Generated Content 
Social Media Analysis for Monitoring Political Sentiment

Social-Based Collaborative Filtering

\section{Social Communities}

\section{Section Editor: Lei Tang and Huan Liu}

Assessing Individual and Group Behavior from Mobility Data: Technological Advances and Emerging Applications

Combining Link and Content for Community Detection

Community Detection and Analysis on Attributed Social Networks

Community Evolution

Competition Within and Between Communities in Social Networks

Connecting Communities

Core Decomposition of Massive, InformationRich Graphs

Demographic, Ethnic, and Socioeconomic Community Structure in Social Networks

Extracting and Inferring Communities via Link Analysis

Geography and Web Communities

Group Representation and Profiling

Scalable Graph Clustering and Its Applications

Small Communities in Social Networks Web Communities Versus Physical Communities

\section{Social Network Analysis}

\section{Section Editor: Przemyslaw Kazienko and} Jarostaw Jankowski

Agent Perspective Social Networks: Distributed Second Degree Estimation

Components of the Network Around an Actor

Computational Trust Models

Crowdsourcing and Social Networks

Distributed Processing of Networked Data

Exchange Networks

Exponential Random Graph Models

Flickr and Twitter Data Analysis

Human Behavior and Social Networks
International Hyperlink Networks

Measurement and Analysis of Online Social

Networks Systems

Motif Analysis

Multilayer Social Networks

Path-Based and Whole-Network Measures

Process of Social Network Analysis

Relational Models

Research Designs for Social Network Analysis

Role Identification of Social Networkers

Scale-Free Nature of Social Networks

Scholarly Network Analysis

Signed Graphs

Similarity Metrics on Social Networks

Social Groups in Crowd

Social Interaction Analysis for Team

Collaboration

Social Web Search

Structural Holes

Twitter Microblog Sentiment Analysis

Virtual Goods in Social Media

Visual Methods and Tools for Social Network Analysis

Wikipedia Knowledge Community

Modeling

\section{Social Network Applications in Business, Organizations, Industry and Case Studies}

\section{Section Editor: Jakob Dijkstra}

Business-to-Business Marketing

Cognitive Strategic Groups

Collection and Analysis of Relational Data from Digital Archives

Collection and Analysis of Relational Data in Organizational and Market Settings

Entrepreneurial Networks

Innovator Networks

Interlocking Directorate Networks

Interorganizational Networks

Intraorganizational Networks

Managerial Networking

Modeling of Business Processes and Crisis Management

Networks of Practice

R\&D Networks 
Social Network Analysis and Organizational

Multimodal Representation

Social Network Analysis in Organizational

Structures Evaluation

Supply Chain Networks

Top Management Team Networks

\section{Social Network Applications in Homeland} Security, Terrorism, Fraud Detection, Public Sector and Case Studies

\section{Section Editor: Rosa M. Benito and Juan Carlos Losada}

Actionable Information in Social Networks, Diffusion of

Android Malware Pattern Recognition for Fraud Detection and Attribution: A Case Study Collaboration Patterns in Software Developer Network

Counterterrorism, Social Network Analysis in Cryptography and Communications Privacy: An Introduction

Dark Side of Online Social Networks: Technical,

Managerial, and Behavioral Perspectives

Disease Surveillance: Case Study

Fraud Detection Using Social Network Analysis:

A Case Study

Legislative Prediction with Political and Social Network Analysis

Mining Blackhole and Volcano Patterns for Fraud

Detection

Misinformation in Social Networks: Analyzing

Twitter During Crisis Events

Role Discovery

Social Collaborative Media in Software

Development

Social Communication Network: Case Study

Social Networks in Emergency Response

Social Networks in Healthcare:

Case Study

Social Order in Online Social Networks

Social Phishing

Spam Detection: E-mail/Social Network

Telecommunications Fraud Detection, Using

Social Networks for

Trust in Social Networks
Social Network Applications in Scientific, Engineering, Medical Domain and Case Studies

\section{Section Editor: Nitin Agarwal and Charalampos Chelmis}

Research on Online Health Communities: A Systematic Review

Social Networking for Open Innovation

Social Networking in the Telecom Industry

Social Networks for Quantified Self

Twitris: A System for Collective Social Intelligence

\section{Social Network Construction, Visualization and Analysis Tools}

\section{Section Editor: Vladimir Batagelj}

Gephi

Guess

NetMiner

Network Data File Formats

NodeXL: Simple Network Analysis for Social Media

ORA: A Toolkit for Dynamic Network Analysis and Visualization

Pajek and PajekXXL

Plug-and-Play Macroscopes: Network Workbench (NWB), Science of Science Tool (Sci2), and Epidemiology Tool (EpiC)

Python Packages for Networks

R Packages for Social Network Analysis

Siena: Statistical Modeling of Longitudinal Network Data

Sources of Network Data

Tools for Networks

Tulip 5

UCINET

\section{Spatio-Temporal Aspects in Social Networks} and Social Media

\section{Section Editor: Gao Cong and Bee-Chung Chen}

Detection of Spatiotemporal Outlier Events in Social Networks 
Location-Based Social Networks

Modeling and Analysis of Spatiotemporal Social

Networks

Social Internet of Mobile Things and Decision

Support Tools

Spatiotemporal Footprints in Social Networks

Spatiotemporal Information for the Web

Spatiotemporal Personalized Recommendation of

Social Media Content

Spatiotemporal Proximity and Social Distance

Spatiotemporal Recommendation in Geo-Social

Networks

Spatiotemporal Topic Detection from Social

Media

Tensor-Based Analysis for Urban Networks

\section{Static Versus Dynamic Networks}

\section{Section Editor: Tansel Ozyer and Ozgur Ulusoy}

Analysis and Visualization of Dynamic Networks

Community Structure Characterization

Detecting and Identifying Communities in

Dynamic and Complex Networks: Definition and Survey

Dynamic Community Detection

Exploring Actor-Level Dynamics in Longitudinal

Networks: The State of the Art

Friends Recommendations in Dynamic Social

Networks

Geotemporal Querying of Social Networks and

Summarization

Influence Propagation in Social Networks with

Positive and Negative Relationships

Instant Messaging for Detecting Dynamic Ego-

Centered Communities
Link Dynamics and Community Formation in Social Networks

Mobile- and Context-Aware Applications of Social Networks

Opinion Diffusion and Analysis on Social Networks

Repository for Multirelational Dynamic Networks

Semantic Social Networks Analysis

Stability and Evolution of Scientific Networks

Temporal Analysis on Static and Dynamic Social Networks Topologies

Time- and Event-Driven Modeling of Blogger Influence

Time-Aware Egocentric Network-Based User Profiling

Tracking Dynamic Community Evolution and Events Mobility in Social Networks

\section{Technology for Online Social Networking and Human Computer Interaction}

\section{Section Editor: H. Ulrich Hoppe and James}

Caverlee

Combining Online Social Networks with Text Analysis

Extracting Rich Social Network Data via the Facebook API

Haptics

Mapping Online Social Media Networks

New Intermediaries of Personal Information: The FB Ecosystem

Patterns in Productive Online Networks: Roles, Interactions, and Communication 


\section{About the Editors}

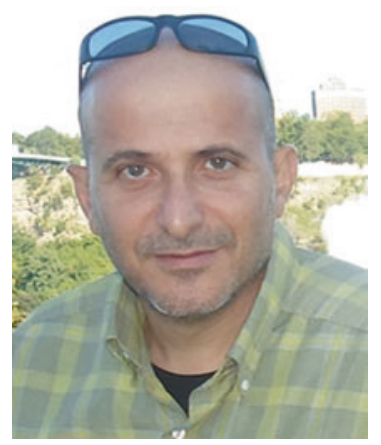

Reda Alhajj received his B.Sc. degree in Computer Engineering with distinction in 1988 from Middle East Technical University, Turkey. Then, he was offered a full scholarship to join the graduate program in Computer Engineering and Information Sciences at Bilkent University, where he received his M.Sc. and Ph.D. degrees with distinction in 1990 and 1993, respectively. Currently, he is tenured professor in the Department of Computer Science at the University of Calgary. He published over 500 papers in refereed international journals and conferences. He served on the program committee of several international conferences. He is founding editor-in-chief of the Springer premier journal Social Networks Analysis and Mining, founding editor-in- chief of the Springer series "Lecture Notes on Social Networks," founding editor-in-chief of the Springer journal Network Modeling Analysis in Health Informatics and Bioinformatics, founding co-editor-in-chief of Springer's Encyclopedia on Social Networks Analysis and Mining, and founding steering chair of the flagship conference IEEE/ACM International Conference on Advances in Social Network Analysis and Mining and of three accompanying symposiums, FOSINT-SI, FAB, and HI-BI-BI. He is member of the editorial boards of the Journal of Information Assurance and Security, Journal of Data Mining and Bioinformatics, and Journal of Data Mining, Modeling and Management; he has been guest editor of a number of special issues and edited a number of conference proceedings. Dr. Alhajj's primary work and research interests are in the areas of computational biology 
and bioinformatics, data mining, social networks, XML, schema integration, and reengineering. He currently leads a large research group of Ph.D. and M.Sc. candidates. He received the Best Graduate Supervision Award from the University of Calgary. Dr. Alhajj recently received, with Dr. Jon Rokne, a donation of equipment valued at $\$ 5$ million from RBC and Teradata for their research on computational intelligence and bioinformatics research. $\mathrm{He}$ recently mentored a number of successful teams, including SANO who ranked first in the Microsoft Imagine Cup competition in Canada and received the KFC Innovation Award in the World Finals held in Russia in July 2013, TRAK who ranked in the top 15 teams in the 2014 Open Data Analysis competition in Canada, and Go2 who ranked first in the Imagine Camp competition organized by Microsoft Canada in March 2014.

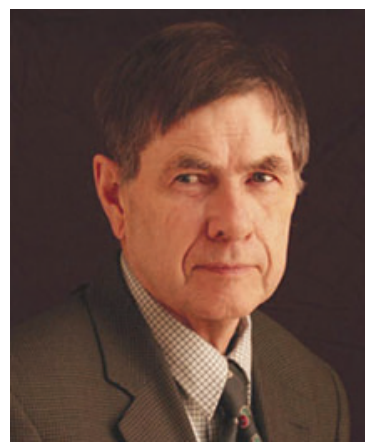

Jon Rokne is a professor in the Department of Computer Science at the University of Calgary. Educated in Norway and Canada, he obtained a Ph.D. in Mathematics from the University of Calgary. He chaired the Computer Science Department at the University of Calgary from 1989 to 1996. He has published extensively in mathematics, including three jointly authored books. His main applied mathematics interest has been in interval analysis, global optimization, and numerical solution of nonlinear equations. In computer science, his focus has been computer graphics, computational geometry, data analysis, social networks, and physically and biologically based computer simulations with publications on leaves, auroras, and ball lightning. He has published one jointly authored computer science book entitled Light Interaction with Plants. In 2003, he organized the Pacific Graphics conference where he jointly edited the proceedings and two special issues of computer graphics journals. He has been an active volunteer with IEEE and has held a number of positions within the organization, including vice-president of publications for IEEE Computer Society and vice-president of publications and board member of IEEE. He spearheaded the three new publications: IEEE Transactions on Cloud Computing, IEEE Cloud Computing Magazine, and IEEE Transactions on Big Data. He chaired the 2013 ASONAM Conference and has been an organizer for other ASONAM conferences. 


\section{Section Editors to Second Edition}

\section{Applied Computational Geometry and Graph Theory for Social Networks}

Richard Chbeir University Pau and Pays Adour, Laboratoire LIUPPA, Anglet, France

Mick J. Ridley University of Bradford, Bradford, UK

\section{Basics of Social Network Model Construction and Evolution}

Ralf Klamma RWTH Aachen, Aachen, Germany

\section{Crowd Sourcing, Opinion Mining, Fusion and Influence Analysis}

Guandong Xu Advanced Analytics Institute, University of Technology, Sydney, Broadway, NSW, Australia

Peng Cui Tsinghua University, Beijing, P.R. China

\section{Current and Future Research Directions}

Andrea Tagarelli University of Calabria, Arcavacata di Rende, Italy

Fabrizio Silvestri Yahoo Inc., London, UK

Data Collection, Data Extraction, and Data Preparation for Social Networks Analysis and Mining

V. S. Subrahmanian Computer Science Department, University of Maryland, College Park, MD, USA

Jeffrey Chan RMIT (Royal Melbourne Institute of Technology), Melbourne, Australia

\section{Data Management for Social Networks and Social Media}

Jaideep Srivastava Department of Computer Science and Engineering, University of Minnesota, Minneapolis, MN, USA

Abdullah Uz Tansel Baruch College, CUNY, New York, USA 


\section{Data Mining and Machine Learning Techniques for Social Networks}

Fakhri Karray Department of Electrical and Computer Engineering, Centre for Pattern Analysis and Machine Intelligence (CPAMI), University of Waterloo, Waterloo, ON, Canada

\section{History of Social Networks: Past, Present and Future}

Talel Abdessalem Telecom ParisTech, Paris, France

Rokia Missaoui Department of Computer Science and Engineering, Université du Québec en Outaouais (UQO), Gatineau, QC, Canada

Internet, Web 2.0, Web Services and Semantic Web

Steffen Staab Institute for Web Science and Technologies, Universität Koblenz-Landau, Koblenz, Germany

Thomas Gottron Institute for Web Science and Technologies, Universität Koblenz-Landau, Koblenz, Germany

Stefan Schlobach YU, Amsterdam, The Netherlands

\section{Linear Algebra and Statistics for Social Networks}

Suheil Khoury American University of Sharjah, Sharjah, United Arab Emirates

\section{Online Social Networks, Social Networking Sites and Social Media}

Irwin King Department of Computer Science and Engineering, The Chinese University of Hong Kong, Shatin, NT, Hong Kong

Jie Tang Department of Computer Science and Technology, Tsinghua University, Beijing, China

\section{Privacy and Security Issues in Social Networks}

Henric Johnson Blekinge Institute of Technology, Karlskrona, Sweden

Felix Wu UC-Davis, Davis, USA

\section{Sentiment Analysis and Recommender Systems}

Giovanni Semeraro University of Bari “Aldo Moro”, Bari, Italy

Cataldo Musto Bari, Italy

\section{Social Communities}

Lei Tang Chief Data Scientist, Clari Inc., Sunnyvale, USA

Huan Liu Data Mining and Machine Learning Lab, School of Computing, Informatics, and Decision Systems Engineering, Arizona State University, Tempe, AZ, USA

\section{Social Network Analysis}

Przemysław Kazienko Department of Computational Intelligence, ENGINE The European Centre for Data Science, Faculty of Computer Science and Management, Wroclaw University of Science and Technology, Wrocław, Poland 
Jaroslaw Jankowski Faculty of Computer Science and Information Technology, West Pomeranian University of Technology, Szczecin, Poland

Social Network Applications in Business, Organizations, Industry and Case Studies

Jacob Dijkstra Department of Sociology, Interuniversity Center for Social Science Theory and Methodology, University of Groningen, Groningen, The Netherlands

Social Network Applications in Homeland Security, Terrorism, Fraud Detection, Public Sector and Case Studies

Rosa M. Benito Universidad Politécnica de Madrid, Madrid, Spain

Juan Carlos Losada Universidad Politécnica de Madrid, Madrid, Spain

Social Network Applications in Scientific, Engineering, Medical Domain and Case Studies

Nitin Agarwal Department of Information Science, University of Arkansas at Little Rock, Little Rock, AR, USA

Charalampos Chelmis University at Albany, Albany, USA

\section{Social Network Construction, Visualization and Analysis Tools}

Vladimir Batagelj Faculty of Mathematics and Physics, Department of Mathematics, University of Ljubljana, Ljubljana, Slovenia

Department of Theoretical Computer Science, Institute of Mathematics, Physics and Mechanics, Ljubljana, Slovenia

University of Primorska, Andrej Marušič Institute, Koper, Slovenia

\section{Static Versus Dynamic Networks}

Tansel Ozyer TOBB Economics and Technology University, Ankara, Turkey

Ozgur Ulusoy Bilkent University, Ankara, Turkey

Technology for Online Social Networking and Human Computer Interaction

H. Ulrich Hoppe Department of Computer Science and Applied Cognitive Science, University of Duisburg-Essen, Duisburg, Germany

James Caverlee Department of Computer Science and Engineering, Texas A\&M University, College Station, TX, USA

\section{Spatio-Temporal Aspects in Social Networks and Social Media}

Gao Cong School of Computer Engineering, Nanyang Technological University, Singapore, Singapore

Bee-Chung Chen LinkedIn, Sunnyvale, CA, USA 


\section{Section Editors to First Edition}

\section{Applied Computational Geometry for Social Networks}

Mick J. Ridley Department of Computing, Bradford University, Bradford, Yorkshire, UK

Daniel Neagu Department of Computing, Bradford University, Bradford, Yorkshire, UK

\section{Basics of Social Network Model Construction and Evolution}

Ralf Klamma RWTH Aachen, Lehrstuhl Informatik 5, Aachen, Germany

\section{Current and Future Research Directions}

Katharina Anna Zweig Department of Computer Science, Complex Network Analysis and Graph Theory, University of Kaiserslautern, Kaiserslautern, Germany

Data Collection, Data Extraction, and Data Preparation for Social Networks Analysis and Mining

V. S. Subrahmanian Computer Science Department, University of Maryland, College Park, MD, USA

\section{Data Management for Social Networks and Social Media}

Jaideep Srivastava Department of Computer Science and Engineering, University of Minnesota, Minneapolis, MN, USA

Abdullah Uz Tansel Baruch College CUNY, New York, NY, USA

\section{Data Mining and Machine Learning Techniques for Social Networks}

Mohamed S. Kamel Department of Electrical and Computer Engineering, Centre for Pattern Analysis and Machine Intelligence (CPAMI), University of Waterloo, Waterloo, ON, Canada

Fakhri Karray Department of Electrical and Computer Engineering, Centre for Pattern Analysis and Machine Intelligence (CPAMI), University of Waterloo, Waterloo, ON, Canada 


\section{Graph Theory for Social Networks (Sociograms)}

Richard Chbeir Laboratoire LIUPPA, University of Pau and Adour Countries, Anglet, France

\section{History of Social Networks: Past, Present and Future}

David Knoke Department of Sociology, University of Minnesota, 909 Social Sciences, Minneapolis, MN, USA

\section{Internet, Web 2.0, Web Services and Semantic Web}

Steffen Staab Institute for Web Science and Technologies, Universität Koblenz-Landau, Koblenz, Germany

Thomas Gottron Institute for Web Science and Technologies, Universität Koblenz-Landau, Koblenz, Germany

\section{Linear Algebra and Statistics for Social Networks}

Suheil Khoury Department of Mathematics and Statistics, American University of Sharjah, Sharjah, United Arab Emirates

\section{Online Social Networks, Social Networking Sites and Social Media}

Irwin King Department of Computer Science and Engineering, The Chinese University of Hong Kong, Shatin, N.T., Hong Kong

Jie Tang Department of Computer Science and Technology, Tsinghua, University, Beijing, China

\section{Privacy and Security Issues in Social Networks}

Roba Abbas University of Wollongong, Wollongong, NSW, Australia

Katina Michael School of Information Systems and Technology, University of Wollongong, Wollongong, NSW, Australia

\section{Social Communities}

Huan Liu Data Mining and Machine Learning Lab, School of Computing, Informatics, and Decision Systems Engineering, Arizona State University, Tempe, AZ, USA

Lei Tang Yahoo-Inc., Advertising Sciences, Yahoo! Labs, Santa Clara, CA, USA

\section{Social Network Analysis}

Przemysław Kazienko Department of Computer Science and Management, Institute of Informatics, Wrocław University of Technology, Wrocław, Poland

Social Network Applications in Business, Organizations, Industry and Case Studies

Rafael Wittek Theoretical Sociology - Department of Sociology, University of Groningen, Groningen, The Netherlands 
Social Network Applications in Homeland Security, Terrorism, Fraud Detection, Public Sector, Politics and Case Studies

Charu C. Aggarwal IBM T. J. Watson Research Center, Yorktown Heights, NY, USA

Hanghang Tong CUNY city college, New York, NY, USA

Social Network Applications in Scientific, Engineering, Medical Domain and Case Studies

Alexandre Passant Galway, Ireland

\section{Social Network Construction, Visualization and Analysis Tools}

Vladimir Batagelj Faculty of Mathematics and Physics, Department of Mathematics, University of Ljubiljana, Ljubijana, Slovenia

\section{Spatio-Temporal Aspects in Social Networks and Social Media}

Ee-Peng Lim School of Information Systems, Singapore Management University, Singapore, Singapore

Hady Wirawan Lauw School of Information Systems, Singapore Management University, Singapore, Singapore

\section{Static Versus Dynamic Networks}

Tansel Ozyer TOBB Economics and Technology University, Ankara, Turkey

Ozgur Ulusoy Department of Computer Engineering Bilkent University, Bilkent, Ankara, Turkey

Technology for Online Social Networking and Human Computer Interaction

Lothar Krempel Max Planck Institute for the Study of Societies, Cologne, Germany 


\section{Advisory Board to Second Edition}

Erol Arkun Ankara, Turkey

Bela Bollobas University of Memphis, Memphis, TN, USA

University of Cambridge, Cambridge, UK

Kathleen M. Carley Carnegie Mellon University, Pittsburgh, PA, USA

Netanomics, Sewickley, PA, USA

Ahmed Elmagarmid Qatar Foundation, Doha, Qatar

John Scott University of Plymouth, Plymouth, UK

Shashi Shekhar Department of Computer Science, University of Minnesota, Minneapolis, MN, USA

Frans Stokman University of Groningen, Groningen, The Netherlands

Leon S. L. Wang National University of Kaohsiung, Taiwan

Dorothea Wagner Karlsruhe Institute of Technology (KIT), Institute of Theoretical Informatics, Karlsruhe, Germany

Barry Wellman University of Toronto, Toronto, ON, Canada

Stanley Wisserman Indiana University, Bloomington, Indiana, USA 


\section{Advisory Board to First Edition}

Erol Arkun Ankara, Turkey

Bela Bollobas University of Memphis and University of Cambridge (England), USA/UK

Kathleen M. Carley Carnegie Mellon University, Pittsburgh, PA, USA

Netanomics, Sewickley, PA, USA

Jack Dongarra University of Tennessee, USA

Ahmed Elmagarmid Qatar Foundation, Qatar

Linton C. Freeman Department of Sociology, School of Social Sciences, University of California, Irvine, CA, USA

Irene H. Frieze University of Pittsburgh, USA

Benjamin H. Gottlieb University of Guelph, Canada

Caroline Haythornthwaite School of Library, Archival and Information Studies, University of British Columbia, Vancouver, BC, Canada

Philippa Eleanor Pattison University of Melbourne, Australia

John Scott University of Plymouth, UK

Shashi Shekhar Department of Computer Science, University of Minnesota, Minneapolis, MN, USA

Dorothea Wagner Karlsruhe Institute of Technology (KIT), Institute of Theoretical Informatics, Karlsruhe, Germany

Barry Wellman University of Toronto, Canada 


\section{Contributors}

Noor Fazilla Abd Yusof Department of Computing Science, University of Aberdeen, Aberdeen, UK

Hervé Abdi School of Behavioral and Brain Sciences, The University of Texas at Dallas, Richardson, TX, USA

Karl Aberer École Polytechnique Fédérale de Lausanne (EPFL), Lausanne, Switzerland

Distributed Inf. Sys Lab., Inst. for Core Computing Science (IIF), EPFL-ICIIF-LSIR, Lausanne, Switzerland

Eytan Adar School of Information and Computer Science and Engineering, University of Michigan, Ann Arbor, MI, USA

Nitin Agarwal Department of Information Science, University of Arkansas at Little Rock, Little Rock, AR, USA

Vinti Agarwal Data Mining, Artificial Intelligence, Information Science, School of Computer and Systems Sciences, Jawaharlal Nehru University, New Delhi, India

Charu C. Aggarwal IBM T. J. Watson Research Center, Yorktown Heights, NY, USA

Karan Aggarwal Department of Computer Science and Engineering, University of Minnesota, Minneapolis, MN, USA

Armen Aghasaryan Alcatel-Lucent Bell Labs, Nozay, France

Rajeev Agrawal Department of Electronics, Computer and Information Technology North Carolina Agricultural and Technical State University, Greensboro, NC, USA

Marlous Agterberg KIN Research, VU University, Amsterdam, The Netherlands

Muhammad Aurangzeb Ahmad Department of Computer Science and Engineering, University of Minnesota, Minneapolis, MN, USA 
Luca Maria Aiello Nokia Bell Labs, Cambridge, UK

Jalel Akaichi Department of Computer Science, King Khaled University Guraiger, Abha, Saudi Arabia

Cuneyt Gurcan Akcora DISTA, Università degli Studi dell'Insubria, Varese, Lombardia, Italy

Agnes Akkerman Nijmegen School of Management, Radboud University, Nijmegen, The Netherlands

Fahimah Al-Awadhi Department of Statistics and Operations Research, Kuwait University, Kuwait City, Kuwait

Omar Alonso Microsoft Corp., Microsoft Corp, Mountain View, CA, USA

Eiman Tamah Al-Shammari College of Computing Science and Engineering, Kuwait University, Kuwait City, Kuwait

Ghufran Al-Shiridah College of Engineering and Petroleum, Kuwait University, Kuwait City, Kuwait

Bernd Amann Sorbonne Universités, UPMC Univ Paris 06, CNRS, Paris, France

Frédéric Amblard IRIT - Université Toulouse 1 Capitole, Toulouse, France

Khaled Ammar University of Waterloo, Waterloo, ON, Canada

Maik Anderka Bad Arolsen, Germany

Paolo Arcaini Charles University, Faculty of Mathematics and Physics, Prague, Czech Republic

Daniel Archambault Department of Computer Science, Swansea University, Swansea, UK

Andreas Artemiou School of Mathematics, Cardiff University, Cardiff, Wales, UK

Martin Atzmueller Tilburg Center for Communication and Cognition, Tilburg University, Tilburg, The Netherlands

Research Center for Information System Design, University of Kassel, Kassel, Germany

David Auber CNRS UMR 5800 LaBRI, University of Bordeaux, Talence, France

Ayman Badawi Department of Mathematics, American University of Sharjah, Sharjah, UAE

David A. Bader School of Computational Science and Engineering, Georgia Institute of Technology, Atlanta, GA, USA

Soojin Bae Cyram Inc., Seoul, South Korea 
James P. Bagrow Mathematics and Statistics and Vermont Complex Systems Center, University of Vermont, Burlington, VT, USA

William Sims Bainbridge Cyber-Human Systems Program, Division of Information and Intelligent Systems, National Science Foundation, HumanCentered Computing, Arlington, VA, USA

Yolanda S. Baker Department of Computer Systems Technology, North Carolina Agricultural and Technical State University, Greensboro, NC, USA

Soumya Banerjee Department of Computer Science and Engineering, Birla Institute of Technology, Mesra, India

Konstantia Barbatsalou University of Coimbra, Coimbra, Portugal

George A. Barnett Department of Communication, University of California, Davis, Davis, USA

Marc Barthelemy Institut de Physique Théorique, CEA, Gif-sur-Yvette, France

Roman Bartusiak Department of Computational Intelligence, ENGINE The European Centre for Data Science, Faculty of Computer Science and Management, Wroclaw University of Science and Technology, Wrocław, Poland

Pierpaolo Basile Department of Computer Science, University of Bari Aldo Moro, Bari, Italy

Valerio Basile Université Côte d'Azur, Inria, CNRS, Sophia Antipolis, France

Vladimir Batagelj Faculty of Mathematics and Physics, Department of Mathematics, University of Ljubljana, Ljubljana, Slovenia

Department of Theoretical Computer Science, Institute of Mathematics, Physics and Mechanics, Ljubljana, Slovenia

University of Primorska, Andrej Marušič Institute, Koper, Slovenia

Luana Batista École de technologie supérieure, Montréal, QC, Canada

Derek Beaton Rotman Research Institute, Baycrest Health Sciences, Toronto, ON, Canada

Alejandro Bellogín Universidad Autónoma de Madrid, Madrid, Spain

Ahmad Bennakhi College of Engineering and Petroleum, Kuwait University, Kuwait City, Kuwait

Michel Béra Centre d'Étude et de Recherche en Informatique et Communications, Conservatoire National des Arts et Métiers, Paris, France

Klaus Berberich Max Planck Institute for Informatics, Saarbrücken, Germany

David Bermbach Information Systems Engineering Research Group, Fakultät IV Elektrotechnik und Informatik, TU Berlin, Berlin, Germany 
Alex Beutel School of Computer Science, Carnegie Mellon University, Pittsburgh, PA, USA

K. K. Bharadwaj Data Mining, Artificial Intelligence, Information Science, School of Computer and Systems Sciences, Jawaharlal Nehru University, New Delhi, India

Yanjie Bian Department of Sociology, University of Minnesota, Minneapolis, MN, USA

Institute for Empirical Social Science Research, Xi'an Jiaotong University, Xi'an, Shaanxi Province, China

Magdalena Bielenia-Grajewska Intercultural Communication and Neurolinguistics Laboratory, Department of Translation Studies, Faculty of Languages, Institute of English, University of Gdansk, Gdansk, Poland

Mareike Bockholt Department of Computer Science, Complex Network Analysis and Graph Theory, University of Kaiserslautern, Kaiserslautern, Germany

Jernej Bodlaj Research, Abelium d.o.o. R\&D, Ljubljana, Slovenia

Petko Bogdanov Department of Computer Science, University of California, Santa Barbara, CA, USA

Francesco Bonchi ISI Foundation, Turin, Italy

Gloria Bordogna CNR IREA - National Research Council, Institute of Electromagnetic Sensing of the Environment, Milan, Italy

Stephen P. Borgatti LINKS Center for Social Network Analysis, Gatton College of Business and Economics, University of Kentucky, Lexington, KY, USA

Katy Börner School of Informatics and Computing, Cyberinfrastructure for Network Science Center, Indiana University, Bloomington, IN, USA

Cristina Bosco Computer Science Department, University of Turin, Turin, Italy

Romain Bourqui CNRS UMR 5800 LaBRI, University of Bordeaux, Talence, France

Makram Bouzid Alcatel-Lucent Bell Labs, Nozay, France

Ross Brennan Hertfordshire Business School, University of Hertfordshire, Hatfield, UK

John Breslin Digital Enterprise Research Institute, National University of Ireland at Galway, Galway, Ireland

Igo Ramalho Brilhante Federal University of Ceara, Fortaleza, Brazil

Piotr Bródka Department of Computational Intelligence, ENGINE - The European Centre for Data Science, Faculty of Computer Science and Management, Wroclaw University of Science and Technology, Wrocław, Poland 
C. Scott Brown Department of Mathematics and Statistics, University of South Alabama, Mobile, AL, USA

Matthias Brücheler Computer Science Department, University of Maryland, College Park, MD, USA

Ivan Brugere University of Illinois at Chicago, Chicago, USA

Jeremy Brunn Ohio Center of Excellence in Knowledge-Enabled Computing (Kno.e.sis), Wright State University, Dayton, OH, USA

Robin Burke College of Computing and Digital Media, DePaul University, Chicago, IL, USA

Karina Cagarman Technical University Berlin, Berlin, Germany

Alberto Caimo Faculty of Economics, University of Lugano, Lugano, Switzerland

Erik Cambria School of Computer Science and Engineering, Nanyang Technological University, Singapore, Singapore

Ricardo Campello Computing Science Department, University of Alberta, Edmonton, AB, Canada

Department of Computer Science, University of São Paulo, São Carlos, SP, Brazil

Marie-Françoise Canut Toulouse Institute of Computer Science Research, University of Toulouse, CNRS, INPT, UPS, UT1, UT2J, Toulouse, France

Systèmes d'Informations Généralisés, Institut de Recherche en Informatique de Toulouse (IRIT), Toulouse, France

Jie Cao Jiangsu Provincial Key Laboratory of E-Business, Nanjing University of Finance and Economics, Nanjing, China

Kathleen M. Carley Carnegie Mellon University, Pittsburgh, PA, USA

Netanomics, Sewickley, PA, USA

Radu C. Cascaval Department of Mathematics, University of Colorado Colorado Springs, Colorado Springs, CO, USA

James Caverlee Department of Computer Science and Engineering, Texas A\&M University, College Station, TX, USA

Rémy Cazabet LIP6, CNRS, Pierre and Marie Curie University, Paris, France

Monika Cerinšek Abelium d.o.o., Research and Development, Ljubljana, Slovenia

Ofelia Cervantes French-Mexican Laboratory of Informatics and Automatic Control (LAFMIA, UMI CNRS 3175), Lyon, France

Sangwhan Cha University of New Brunswick, People in Motion Lab, Fredericton, Canada 
Roshni Chakraborty Indian Institute of Technology, Patna, Bihar, India

Raymond H. Chan Department of Mathematics, The Chinese University of Hong Kong, Shatin, NT Hong Kong SAR, China

Joydeep Chandra Indian Institute of Technology, Patna, Bihar, India

Kuiyu Chang LinkSure China Holding, Singapore, Singapore

Ayona Chatterjee Department of Statistics and Biostatistics, California State University, East Bay, Hayward, CA, USA

Iti Chaturvedi School of Computer Science and Engineering, Nanyang Technological University, Singapore, Singapore

Duen Horng Chau College of Computing, Georgia Institute of Technology, Atlanta, GA, USA

Michael Chau School of Business, Faculty of Business and Economics, The University of Hong Kong, Hong Kong SAR, China

Nitesh V. Chawla Department of Computer Science and Engineering, University of Notre Dame, Notre Dame, IN, USA

Interdisciplinary Center for Network Science and Applications (iCeNSA), Notre Dame, IN, USA

Sanjay Chawla School of Information Technologies, University of Sydney, Sydney, NSW, Australia

Richard Chbeir University Pau and Pays Adour, Laboratoire LIUPPA, Anglet, France

Bee-Chung Chen LinkedIn, Sunnyvale, CA, USA

Enhong Chen School of Computer Science and Technology, University of Science and Technology of China, Anhui, China

Che-Man Cheng Department of Mathematics, University of Macau, Macau, China

Zhiyuan Cheng Department of Computer Science and Engineering, Texas A\&M University, College Station, TX, USA

Adrian David Cheok Imagineering Institute, Iskandar Puteri, Malaysia

City, University of London, London, UK

Sean Chester Department of Computer Science, Norwegian University of Science and Technology, Trondheim, Norway

Yun Chi NEC Laboratories America, Inc., Cupertino, CA, USA

Antonio M. Chiesi Department of Social and Political Sciences, Università degli Studi di Milano, Milan, Italy

Chieh-Li Chin School of Information Sciences, University of Illinois at Urbana-Champaign, Champaign, IL, USA 
Woosik Choi Cyram Inc., Seoul, South Korea

Sarvenaz Choobdar CRACS and INESC-TEC, Faculdade de Ciencias, Universidade do Porto, Porto, Portugal

Nazim Choudhury Complex Systems Research Group Faculty of Engineering and IT, The University of Sydney, Sydney, NSW, Australia

Vincent Chua Department of Sociology, National University of Singapore, Singapore, Singapore

Chung Joo Chung Department of Journalism and Mass Communication, Kyungpook National University, Daegu, Korea

Kon Shing Kenneth Chung Complex Systems Research Group, Project Management Program, Faculty of Engineering and Information Technologies, School of Civil Engineering, J05, The University of Sydney, Sydney, NSW, Australia

Kevin D. Clark Villanova School of Business, Villanova University, Villanova, PA, USA

Germà Coenders Department of Economics, University of Girona, Girona, Spain

Mauro Coletto IMT School for Advanced Studies, Ca' Foscari University of Venice, Pisa, Italy

Kathleen M. T. Collins Department of Curriculum and Instruction, University of Arkansas at Fayetteville, Fayetteville, AR, USA

Gao Cong School of Computer Engineering, Nanyang Technological University, Singapore, Singapore

Lluís Coromina Department of Economics, University of Girona, Girona, Spain

Carlos D. Correa Lawrence Livermore National Laboratory, Center for Applied Scientific Computing, Livermore, CA, USA

Michele Coscia Center for International Development (CID), Harvard Kennedy School, Cambridge, MA, USA

Blaise Cronin School of Informatics and Computing, Indiana University, Bloomington, IN, USA

Bin Cui Key Lab of High Confidence Software Technologies (MOE), School of EECS, Peking University, Beijing, China

Anwitaman Datta Nanyang Technological University, Singapore, Singapore

Marco de Gemmis Department of Computer Science, University of Bari Aldo Moro, Bari, Italy

Maylis Delest CNRS UMR 5800 LaBRI, University of Bordeaux, Talence, France 
Sergio de los Santos ElevenPaths, Malaga, Spain

Narsingh Deo Department of EECS, Univ of Central Florida, Orlando, FL, USA

David DeRoure Oxford e-Research Centre, University of Oxford, Oxford, UK

Mikael Desertot LAMIH UMR CNRS/UVHC 8201, University Lille North of France, Valenciennes, France

Amol Deshpande Department of Computer Science, University of Maryland, College Park, MD, USA

Prasanna Desikan Division of Applied Research, Allina Health Hospitals and Clinics, Minneapolis, MN, USA

Rajarshi Dey Department of Mathematics and Statistics, University of South Alabama, Mobile, AL, USA

Zeineb Dhouioui Department of Computer Science, Bestmod Laboratory, ISG Tunis Université de Tunis, Tunis, Tunisia

Jana Diesner School of Information Sciences, University of Illinois at Urbana-Champaign, Champaign, IL, USA

Dominic DiFranzo Department of Electronics and Computer Science, University of Southampton, Southampton, UK

Jacob Dijkstra Department of Sociology, Interuniversity Center for Social Science Theory and Methodology, University of Groningen, Groningen, The Netherlands

Ying Ding School of Informatics and Computing, Indiana University, Bloomington, IN, USA

Tommaso Di Noia SisInf Lab, Polytechnic University of Bari, Bari, Italy

Sotiris Diplaris Centre for Research and Technology Hellas, Information Technologies Institute, Thessaloniki, Greece

Pramod Divakamurthy BioComplex Laboratory, Department of Computer Sciences, School of Computing, Florida Institute of Technology, Melbourne, FL, USA

Carlotta Domeniconi Department of Computer Science, George Mason University, Fairfax, VA, USA

Cailing Dong Department of Information Systems, University of Maryland, Baltimore County, Baltimore, MD, USA

Michelle Donnell Department of Electronics, Computer and Information Technology North Carolina Agricultural and Technical State University, Greensboro, NC, USA

Patrick Doreian Faculty of Social Sciences, University of Ljubljana, Ljubljana, Slovenia

Department of Sociology, University of Pittsburgh, Pittsburgh, PA, USA 
Jonathan Dubois CNRS UMR 5800 LaBRI, University of Bordeaux, Talence, France

Schahram Dustdar Distributed Systems Group, Institute for Information Systems, TU Wien, Vienna, Austria

Catherine Dwyer Seidenberg School of Computer Science and Information Systems, Pace University, New York, NY, USA

Bosede Iyiade Edwards Imagineering Institute, Iskandar Puteri, Malaysia City, University of London, London, UK

Dmitry Efimov Faculty of Mechanics and Mathematics, Moscow State University, Moscow, Russia

Ahmed Elbagoury University of Waterloo, Waterloo, ON, Canada

Tina Eliassi-Rad College of Computer and Information Science, Northeastern University, Boston, MA, USA

Yuval Elovici Department of Information Systems Engineering, Ben-Gurion University of the Negev, Beer-Sheva, Israel

Muhammad El-Taha Department of Mathematics and Statistics, University of Southern Maine, Portland, ME, USA

Aida Eslami Centre for Heart Lung Innovation, University of British Columbia, Vancouver, BC, Canada

Martin Ester School of Computing Science, Simon Fraser University, Burnaby, BC, Canada

Michael R. Evans Department of Computer Science, University of Minnesota, Minneapolis, MN, USA

Martin G. Everett Mitchell Centre for Social Network Analysis, School of Social Science, University of Manchester, Manchester, UK

Davide Eynard Faculty of Informatics, Institute of Computational Sciences, Università della Svizzera Italiana, Lugano, Switzerland

Justin Fagnan Computing Science Department, University of Alberta, Edmonton, AB, Canada

Christos Faloutsos School of Computer Science, Carnegie Mellon University, Pittsburgh, PA, USA

Josemar Faustino da Cruz BioComplex Laboratory, Department of Computer Sciences, School of Computing, Florida Institute of Technology, Melbourne, FL, USA

Shihui Feng Information Management, Division of Information and Technology Studies, University of Hong Kong, Hong Kong, China

Zhuo Feng AI+R, Microsoft, Sunnyvale, CA, USA 
Emilio Ferrara Center for Complex Networks and Systems Research, School of Informatics and Computing, Indiana University, Bloomington, IN, USA

Elena Ferrari DISTA, Università degli Studi dell'Insubria, Varese, Lombardia, Italy

Fernando Ferri Institute of Research on Population and Social Policies IRPPS, National Research Council -CNR, Rome, Italy

Linton C. Freeman Department of Sociology, School of Social Sciences, University of California, Irvine, CA, USA

Nial Friel INSIGHT: The National Centre for Big Data Analytics, School of Mathematical Sciences, University College Dublin, Dublin, Ireland

Agata Fronczak Faculty of Physics, Warsaw University of Technology, Warsaw, Poland

Piotr Fronczak Faculty of Physics, Warsaw University of Technology, Warsaw, Poland

Eugenia Galeota Computational Epigenetics Group, Center for Genomic Science IIT@SEMM, Istituto Italiano di Tecnologia, Milano, Italy

G. R. Gangadharan Institute for Development and Research in Banking Technology (IDRBT), Hyderabad, India

Steven S. Garcia Department of Computer Science, University of New Mexico, Albuquerque, NM, USA

Fabio Gasparetti Department of Engineering, Roma Tre University, Rome, Italy

Fatih Gedikli Department of Computer Science, TU Dortmund, Dortmund, Germany

Ghi-Hoon Ghim Cyram Inc., Seoul, South Korea

Fosca Giannotti KDDLab, ISTI-CNR, Pisa, Italy

Paolo Giordani Department of Statistical Sciences, Sapienza University of Rome, Rome, Italy

Argyro Gkorogia Department of Information and Communication Systems Engineering, University of the Aegean, Karlovassi, Samos, Greece

Peter Gloor Center for Collective Intelligence, MIT, Cambridge, MA, USA

Laurent-Walter Goix Telecom Italia S.p.A, Milan, MI, Italy

Mark Goldberg Department of Computer Science, Rensselaer Polytechnic Institute, Troy, NY, USA

Isabella Gollini Department of Civil Engineering, University of Bristol, Bristol, UK

Tim Gollub Bauhaus-Universität Weimar, Weimar, Germany 
Paulo Gonçalves Faculty of Economics, Institute of Management, Università della Svizzera Italiana (USI), Lugano, Switzerland

Michael F. Goodchild Department of Geography, University of California Santa Barbara, Santa Barbara, CA, USA

Sebastian Gottifredi Institute for Research in Computer Science and Engineering (ICIC), UNS - CONICET, Bahia Blanca, Argentina

Rick Grannis Department of Sociology, UCLA, Los Angeles, CA, USA

Sociology, UCI, Irvine, CA, USA

Kshanti A. Greene Wily Desert Labs, Gardiner, ME, USA

Management Sciences Inc, Albuquerque, NM, USA

Patrizia Grifoni Institute of Research on Population and Social Policies IRPPS, National Research Council -CNR, Rome, Italy

Anatoliy Gruzd Ted Rogers School of Management, Ryerson University, Toronto, ON, Canada

Vincent Guillemot Bioinformatics and Biostatistics Hub, Institut Pasteur (IP), C3BI, USR 3756 CNRS, Paris, France

Basak Guler Department of Electrical Engineering, The Pennsylvania State University, State College, PA, USA

Francesco Gullo UniCredit, R\&D Dept, Rome, Italy

Pritam Gundecha IBM Research, Almaden, San Jose, CA, USA

Venkata M. V. Gunturi IIIT-Delhi, New Delhi, India

Aditi Gupta Indraprastha Institute of Information Technology, Delhi, New Delhi, India

Claudio Gutierrez Department of Computer Science, Universidad de Chile, Santiago, Chile

Antonio Guzmán ElevenPaths, Malaga, Spain

Tiziana Guzzo Institute of Research on Population and Social Policies IRPPS, National Research Council -CNR, Rome, Italy

Matthias Hagen Bauhaus-Universität Weimar, Weimar, Germany

Zhen Hai Institute for Infocomm Research, Singapore, Singapore

Juho Hamari Game Research Lab, UC Pori, Tampere University of Technology, Tampere, Finland

UC Pori, University of Turku, Turku, Finland

Abdelwahab Hamou-Lhadj Department of Electrical and Computer Engineering, Concordia University, Montreal, QC, Canada

Jiawei Han Department of Computer Science, University of Illinois at Urbana-Champaign, Urbana, IL, USA 
Jo Hanisch Strategic Information Management Group, School of Computer and Information Science, University of South Australia, Adelaide, SA, Australia

Ward Hanson Department of Economics, Stanford University, Stanford, CA, USA

Mariann Hardey Institute for Advanced Research in Computing (iARC), University of Durham, Durham, UK

Paul Haridakis School of Communication Studies, Kent State University, Kent, OH, USA

Mostafa M. Hassan Sandvine Inc., Waterloo, ON, Canada

Caroline Haythornthwaite School of Information Studies, Syracuse University, Syracuse, NY, USA

Jingrui He Computer Science and Engineering, Arizona State University, Tempe, AZ, USA

Yulan He School of Engineering and Applied Science, Aston University, Birmingham, UK

Tobias Hecking Department of Computer Science and Applied Cognitive Science, University of Duisburg-Essen, Duisburg, Germany

Eelke M. Heemskerk Department of Political Science, University of Amsterdam, Amsterdam, The Netherlands

Denis Helic Knowledge Management Institute, Graz University of Technology, Graz, Austria

James A. Hendler Department of Computer Science, Rensselaer Polytechnic Institute, Troy, NY, USA

Computer and Cognitive Science Departments, Tetherless World Constellation, Rensselaer Polytechnic Institute, Troy, NY, USA

Sébastien Heymann LIP6, Université Pierre et Marie Curie (UPMC), Paris, France

Jan Hidders Faculty of Electrical Engineering, Mathematics and Computer Science Delft University of Technology, Delft, The Netherlands

Pascal Hitzler Data Semantics Laboratory, Department of Computer Science and Engineering, Wright State University, Dayton, OH, USA

Valentina Hlebec Faculty of Social Sciences, University of Ljubljana, Ljubljana, Slovenia

Myron Hlynka Department of Mathematics and Statistics, University of Windsor, Windsor, ON, Canada 
Jörg Hoffmann Department of Computer Science, Saarland University, Saarbrücken, Germany

Steven C. H. Hoi School of Information Systems, Singapore Management University, Singapore, Singapore

Petter Holme Department of Energy Science, Sungkyunkwan University, Suwon, Korea

IceLab, Department of Physics, Umeå University, Umeå, Sweden

H. Ulrich Hoppe Department of Computer Science and Applied Cognitive Science, University of Duisburg-Essen, Duisburg, Germany

Emőke-Agnes Horvat Interdisciplinary Center for Scientific Computing (IWR), University of Heidelberg, Heidelberg, Germany

Department of Computer Science, Complex Network Analysis and Graph Theory, University of Kaiserslautern, Kaiserslautern, Germany

Liaquat Hossain Information Management, Division of Information and Technology Studies, University of Hong Kong, Hong Kong, China

Complex Systems Research Group, Project Management Program, Faculty of Engineering and Information Technologies, The University of Sydney, Sydney, NSW, Australia

Andreas Hotho Computational Social Science Group, GESIS - Leibniz Institute for the Social Sciences, Cologne, Germany

Hsun-Ping Hsieh Department of Electrical Engineering, National Cheng Kung University (NCKU), Tainan, Taiwan

Bo Hu School of Computing Science, Simon Fraser University, Burnaby, BC, Canada

Xia Hu Department of Computer Science and Engineering, Texas A\&M University, College Station, TX, USA

Yifan Hu Yahoo Research, Yahoo Inc, New York, NY, USA

Cindy Hui Department of Industrial and Systems Engineering, Rensselaer Polytechnic Institute, Troy, NY, USA

Mark Huisman Department of Sociology/ICS, University of Groningen, Groningen, The Netherlands

Edward Hung The Department of Computing, The Hong Kong Polytechnic University, Hung Hom, Hong Kong

Mohd Heikal Husin Service Computing, School of Computer Sciences, Universiti Sains Malaysia, Gelugor, Pulau Pinang, Malaysia

Rania Ibrahim University of Waterloo, Waterloo, ON, Canada 
Ken'ichi Ikeda Department of Media Studies, Doshisha University, Kyoto, Japan

Wojciech Indyk Department of Computational Intelligence, ENGINE - The European Centre for Data Science, Faculty of Computer Science and Management, Wroclaw University of Science and Technology, Wrocław, Poland

Ashutosh Jadhav Ohio Center of Excellence in Knowledge-Enabled Computing (Kno.e.sis), Wright State University, Dayton, OH, USA

IBM Research, Yorktown Heights, NY, USA

Manfred Jaeger Department of Computer Science, Aalborg University, Aalborg, Denmark

Mohsen Jamali School of Computing Science, Simon Fraser University, Burnaby, BC, Canada

Jarosław Jankowski Faculty of Computer Science and Information Technology, West Pomeranian University of Technology, Szczecin, Poland

Michal Jankowski-Lorek Faculty of Informatics, Polish-Japanese Institute of Information Technology, Warsaw, Poland

Dietmar Jannach Department of Computer Science, TU Dortmund, Dortmund, Germany

Marco Alberto Javarone University of Hertfordshire, Hatfield, UK

Kent Wickstrøm Jensen Department of Entrepreneurship and Relationship Management, University of Southern Denmark, Kolding, Denmark

Meng Jiang Department of Computer Science and Engineering, University of Notre Dame, Notre Dame, IN, USA

Peiquan Jin School of Computer Science and Technology, University of Science and Technology of China, Hefei, China

Rong Jin Department of Computer Science and Engineering, Michigan State University, East Lansing, MI, USA

Xiao-Qing Jin Department of Mathematics, University of Macau, Macau, China

Anna Johnston Salinger Privacy, Sydney, NSW, Australia

Isaac Jones CIDSE, Arizona State University, Tempe, AZ, USA

Krzysztof Juszczyszyn Institute of Computer Science, Wroclaw University of Technology, Wroclaw, Poland

Lisa Kaati Department of Information Technology, Uppsala University, Uppsala, Sweden

Charles Kadushin Department of Sociology, Cohen Center for Modern Jewish Studies, Brandeis University, Waltham, MA, USA 
Tomasz Kajdanowicz Faculty of Computer Science and Management, Department of Computational Intelligence, Wroclaw University of Science and Technology, Wrocław, Poland

Andreas Kaltenbrunner Eurecat, Barcelona, Spain

Mohamed S. Kamel Department of Electrical and Computer Engineering, Centre for Pattern Analysis and Machine Intelligence (CPAMI), University of Waterloo, Waterloo, ON, Canada

Pavan Kapanipathi Ohio Center of Excellence in Knowledge-Enabled Computing (Kno.e.sis), Wright State University, Dayton, OH, USA

IBM Research, Yorktown Heights, NY, USA

Andreas M. Kaplan Department of Marketing, ESCP Europe Business School, Paris, France

Komal Kapoor Department of Computer Science and Engineering, University of Minnesota, Minneapolis, MN, USA

Andrea Kappes Institute of Theoretical Informatics, Karlsruhe Institute of Technology (KIT), Karlsruhe, Germany

Bruce M. Kapron Department of Computer Science, University of Victoria, Victoria, BC, Canada

Panagiotis Karampelas Department of Informatics and Computers, Hellenic Air Force Academy, Dekelia, Greece

Fakhri Karray Department of Electrical and Computer Engineering, Centre for Pattern Analysis and Machine Intelligence (CPAMI), University of Waterloo, Waterloo, ON, Canada

Andrea L. Kavanaugh Center for Human-Computer Interaction, Virginia Polytechnic Institute and State University (Virginia Tech), Blacksburg, VA, USA

Przemysław Kazienko Department of Computational Intelligence, ENGINE The European Centre for Data Science, Faculty of Computer Science and Management, Wroclaw University of Science and Technology, Wrocław, Poland

Brian C. Keegan University of Colorado, Boulder, CO, USA

Alexandros Kefallonitis Department of Information and Communication Systems Engineering, University of the Aegean, Karlovassi, Samos, Greece

Hajer Kefi CEDAG, University Paris Descartes, Paris, France

School of Computing, National University of Singapore, Singapore, Singapore

Patrick Kenis Institute for Public Management and Governance, WU Vienna University of Economics and Business, Vienna, Austria

Department of Organization Studies, Tilburg University, Tilburg, The Netherlands 
Raphaël Khoury Departement of Computer Science and Mathematics, Université du Québec à Chicoutimi, Chicoutimi, QC, Canada

Richard Khoury Department of Computer Science and Software Engineering, Université Laval, Québec City, QC, Canada

Michael Kifer Department of Computer Science, Stony Brook University, Stony Brook, NY, USA

Kangmin Kim Cyram Inc., Seoul, South Korea

Irwin King Department of Computer Science and Engineering, The Chinese University of Hong Kong, Shatin, NT, Hong Kong

Masaru Kitsuregawa Institute of Industrial Science, University of Tokyo, Tokyo, Meguro Ku, Japan

Joseph M. Kizza Department of Computer Science and Engineering, The University of Tennessee-Chattanooga, Chattanooga, TN, USA

Matthias Klusch German Research Center for Artificial, Intelligence (DFKI), Saarbruecken, Germany

Joe M. Kniss Department of Computer Science, University of New Mexico, Albuquerque, NM, USA

David Knoke Department of Sociology, University of Minnesota, Minneapolis, MN, USA

Matthias Knorr NOVA LINCS and Dept. of Computer Science, Faculdade de Ciências e Tecnologia, Universidade NOVA de Lisboa, Caparica, Portugal

Youngjin Ko Cyram Inc., Seoul, South Korea

Tina Kogovšek Faculty of Arts and Faculty of Social Sciences, University of Ljubljana, Ljubljana, Slovenia

Eric D. Kolaczyk Department of Mathematics and Statistics, Boston University, Boston, MA, USA

Georgia Koloniari Department of Applied Informatics, University of Macedonia, Thessaloniki, Greece

Ioannis Kompatsiaris Centre for Research and Technology Hellas, Information Technologies Institute, Thessaloniki, Greece

Haridimos Kondylakis Department of Electrical Engineering and Computer Science, Leibniz University Hannover, Hannover, Germany

Foundation for Research and Technology Hellas, Institute of Computer Science (ICS), Hellas, Greece

Xiangnan Kong Department of Computer Science, University of Illinois at Chicago, Chicago, IL, USA

Dimitre Davidov Kostadinov Alcatel-Lucent Bell Labs, Nozay, France 
M. Krész Institute of Applied Sciences, University of Szeged, Szeged, Hungary

Jan Kratzer Technical University Berlin, Berlin, Germany

Robert W. Krause Department of Sociology/ICS, University of Groningen, Groningen, The Netherlands

Verena Kreilinger Unified Theory of Information Research Group (UTI), Vienna, Austria

Lothar Krempel Max Planck Institute for the Study of Societies, Cologne, Germany

Adila Krisnadhi Department of Computer Science and Engineering, Wright State University, Dayton, OH, USA

Faculty of Computer Science, Universitas Indonesia, Depok, Indonesia

Krzysztof Kulakowski Faculty of Physics and Applied Computer Science, Department of Applied Informatics and Computational Physics, AGH University of Science and Technology, Kraków, Poland

Cherukuri Aswani Kumar School of Information Technology and Engineering, VIT University, Vellore, India

Ponnurangam Kumaraguru Indraprastha Institute of Information Technology, Delhi, New Delhi, India

Jérôme Kunegis Institute for Web Science and Technologies, University of Koblenz-Landau, Koblenz, Germany

Tsung-Ting Kuo UCSD Health Department of Biomedical Informatics, University of California San Diego, La Jolla, CA, USA

V. Labatut Laboratoire Informatique d'Avignon (LIA EA 4128), University of Avignon, Avignon, France

Alona Labun Jeugdhulp Friesland, Leeuwarden, The Netherlands

Antoine Lambert Inria Centre de recherche de Paris, Paris, France

Christine Largeron Univ. Lyon, UJM-Saint-Etienne,CNRS, Laboratoire Hubert Curien UMR 5516, Saint Etienne, France

Philippe Laublet Université Paris-Sorbonne, Paris, France

Matthew Lease School of Information, University of Texas at Austin, Austin, TX, USA

Sylvain Lecomte LAMIH UMR CNRS/UVHC 8201, University Lille North of France, Valenciennes, France

Joo Young Lee Department of Computer Science, Innopolis University, Innopolis, Russia

Wang-Chien Lee Department of Computer Science and Engineering, Pennsylvania State University, University Park, PA, USA 
Nancy L. Leech School of Education and Human Development, University of Colorado Denver, Denver, CO, USA

Roger Leenders Department of Organization Studies, Tilburg School of Social and Behavioral Sciences, Tilburg University, Tilburg, The Netherlands

Bénédicte LeGrand Centre de Recherche en Informatique, Paris, France

Sune Lehmann DTU Compute, Technical University of Denmark, Kongens Lyngby, Denmark

Jürgen Lerner Department of Computer and Information Science, University of Konstanz, Constance, Germany

Carson K. Leung Department of Computer Science, University of Manitoba, Winnipeg, MB, Canada

Kevin Lewis Department of Sociology, University of California, San Diego, La Jolla, CA, USA

Michael S. Lewis-Beck Department of Political Science, University of Iowa, Iowa City, IA, USA

Cheng-Te Li Department of Statistics, National Cheng Kung University (NCKU), Tainan, Taiwan

Guangxia Li School of Computer Science and Technology, Xidian University, Xi'an, China

Lei Li School of Computer Science and Information Technology, Hefei University of Technology, Hefei, Anhui, China

Linna Li Department of Geography, California State University Long Beach, Long Beach, CA, USA

Tim M. H. Li Department of Paediatrics and Adolescent Medicine, LKS Faculty of Medicine, The University of Hong Kong, Hong Kong SAR, China

Zhongmou Li Department of Management Science and Information Systems, Rutgers the State University of New Jersey, Newark, NJ, USA

Bin Liang School of Computer Science, Fudan University, Shanghai, China

Susanna Liberti Department of Biology, University of Rome Tor Vergata, Rome, Italy

Ee-Peng Lim School of Information Systems, Singapore Management University, Singapore, Singapore

Chenghua Lin Department of Computing Science, University of Aberdeen, Aberdeen, UK

Sheng Lin School of Computer Science and Technology, University of Science and Technology of China, Hefei, China

Shou-De Lin Department of Computer Science and Information Engineering, National Taiwan University (NCKU), Taipei, Taiwan 
Yu-Ru Lin School of Information Sciences, University of Pittsburgh, Pittsburgh, PA, USA

Panagiotis Lionakis Department of Computer Science, University of Crete, Heraklion, Greece

Bing Liu Department of Computer Science, University of Illinois at Chicago, Chicago, IL, USA

Huan Liu Data Mining and Machine Learning Lab, School of Computing, Informatics, and Decision Systems Engineering, Arizona State University, Tempe, AZ, USA

Ninghao Liu Department of Computer Science and Engineering, Texas A\&M University, College Station, TX, USA

Qi Liu School of Computer Science and Technology, University of Science and Technology of China, Anhui, China

School of Computer Science, Fudan University, Shanghai, China

Xin Liu École Polytechnique Fédérale de Lausanne (EPFL), Lausanne, Switzerland

David Lo School of Information Systems, Singapore Management University, Singapore, Singapore

Pasquale Lops Department of Computer Science, University of Bari Aldo Moro, Bari, Italy

Karampelas Loukas Department of Information and Communication Systems Engineering, University of the Aegean, Karlovassi, Samos, Greece

Chen Lu Ohio Center of Excellence in Knowledge-Enabled Computing (Kno.e.sis), Wright State University, Dayton, OH, USA

LinkedIn, Mountain View, CA, USA

Jian Lu State Key Laboratory for Novel Software Technology, Nanjing University, Nanjing, Jiangsu, China

Claudio Lucchese Ca' Foscari University of Venice, Pisa, Italy

Giuseppe Lugano ERAdiate Team, University of Žilina, Žilina, Slovakia

Michael R. Lyu Department of Computer Science and Engineering, The Chinese University of Hong Kong, Shatin, NT, Hong Kong

Hao Ma Microsoft Research, Redmond, WA, USA

Jose Antonio Macedo Federal University of Ceara, Fortaleza, Brazil

Kevin Macnish Inter-Disciplinary Ethics Applied Centre, University of Leeds, Leeds, UK

Malik Magdon-Ismail Department of Computer Science, Rensselaer Polytechnic Institute, Troy, NY, USA 
Patrick G. Maggitti Villanova School of Business, Villanova University, Villanova, PA, USA

Matteo Magnani Department of Information Technology, Uppsala University, Uppsala, Sweden

Khaled Mahdi College of Engineering and Petroleum, Kuwait University, Kuwait City, Kuwait

Charles Mainhart Department of Computer Systems Technology, North Carolina Agricultural and Technical State University, Greensboro, NC, USA

Roger F. Malina School of Arts, Technology, and Emerging Communication, The University of Texas at Dallas, Richardson, TX, USA

Janet Mann Department of Biology and Psychology, Georgetown University, Washington, DC, USA

Andrew Marchese Mathematics Department, University of Tennessee, Knoxville, TN, USA

Pierre Maret UMR 5516 Laboratoire Hubert Curien, Université de Lyon, CNRS, Saint- Étienne, France

Vasileios Maroulas Mathematics Department, University of Tennessee, Knoxville, TN, USA

Ludger Martin Faculty of Design - Computer Science-Media, Hochschule RheinMain, Wiesbaden, Germany

Maria Vanina Martinez Institute for Research in Computer Science and Engineering (ICIC), UNS - CONICET, Bahia Blanca, Argentina

Patrick Mary CNRS UMR 5800 LaBRI, University of Bordeaux, Talence, France

Moreno Marzolla Department of Computer Science and Engineering, University of Bologna, Bologna, Italy

Pablo Mateos CIESAS Research Center, Guadalajara, Mexico

Maristella Matera Dipartimento di Elettronica, Informazione e Bioingegneria, Politecnico di Milano, Milan, Italy

Morgan Mathiaut CNRS UMR 5800 LaBRI, University of Bordeaux, Talence, France

Takazumi Matsumoto Okinawa Institute of Science and Technology, Okinawa, Japan

Matteo Matteucci Department of Electronics Information and Bioengineering, Politecnico di Milano, Milan, Italy

Ian McCulloh Applied Physics Laboratory, Johns Hopkins University, Laurel, MD, USA 
Edward McFowland III Event and Pattern Detection Laboratory, H. J. Heinz III College, Carnegie Mellon University, Pittsburgh, PA, USA

Patrick J. McSweeney Department of Electrical Engineering and Computer Science, Syracuse University, Syracuse, NY, USA

Farshid Mehrdoust Department of Applied Mathematics, Faculty of Mathematical Sciences, University of Guilan, Rasht, Iran

Kishan Mehrotra Department of Electrical Engineering and Computer Science, Syracuse University, Syracuse, NY, USA

Isabel Meirelles Faculty of Design, OCAD University, Toronto, ON, Canada

Guy Melançon CNRS UMR 5800 LaBRI, University of Bordeaux, Talence, France

Ronaldo Menezes BioComplex Laboratory, Department of Computer Sciences, School of Computing, Florida Institute of Technology, Melbourne, FL, USA

Henning Meyerhenke Institute of Theoretical Informatics, Karlsruhe Institute of Technology (KIT), Karlsruhe, Germany

Alessandro Micarelli Department of Engineering, Roma Tre University, Rome, Italy

Katina Michael School of Information Systems and Technology, University of Wollongong, Wollongong, NSW, Australia

Radosław Michalski Department of Computational Intelligence, ENGINE The European Centre for Data Science, Wroclaw University of Science and Technology, Wrocław, Poland

Jacob L. Miller Department of Management, LeBow College of Business, Drexel University, Philadelphia, PA, USA

Peter Miller School of Psychology, Deakin University, Geelong, VIC, Australia

Rokia Missaoui Department of Computer Science and Engineering, Université du Québec en Outaouais (UQO), Gatineau, QC, Canada

Ahmed Ould Mohamed Moctar Department of Mathematics and Computer Science, Cheikh Anta Diop University, Dakar-Fann, Senegal

John W. Mohr Department of Sociology, University of California, Santa Barbara, CA, USA

Aleksandra Mojsilović Mathematical Sciences Department, IBM Thomas J. Watson Research Center, Yorktown Heights, NY, USA

Jayanta Mondal Microsoft Corporation, Redmond, WA, USA

Fabio Luciano Mondin Telecom Italia S.p.A, Torino, TO, Italy 
Juan L. Moreno Dickson Poon School of Law, King's College London, London, UK

Mikołaj Morzy Institute of Computing Science, Poznan University of Technology, Poznań, Poland

Andrej Mrvar Faculty of Social Sciences, University of Ljubljana, Ljubljana, Slovenia

Chris Muelder Computer Science Department, University of California at Davis, Davis, CA, USA

Fabrice Muhlenbach Univ. Lyon, UJM-Saint-Etienne,CNRS, Laboratoire Hubert Curien UMR 5516, Saint Etienne, France

Subhabrata Mukherjee Department of Databases and Information Systems, Max-Planck-Institut für Informatik, Saarbrücken, Germany

Madhuri S. Mulekar Department of Mathematics and Statistics, University of South Alabama, Mobile, AL, USA

Idris Oladele Muniru Imagineering Institute, Iskandar Puteri, Malaysia City, University of London, London, UK

Department of Computer Science, Universiti Teknologi Malaysia, Skudai, Johor, Malaysia

Ethan V. Munson Department of EECS, University of Wisconsin-Milwaukee, Milwaukee, WI, USA

Saman Musacchio Université Paris-Sorbonne, Paris, France

Katarzyna Musial Department of Informatics, King's College London, London, UK

Department of Computing and Informatics, Bournemouth University, Poole, UK

Preslav Nakov Qatar Computing Research Institute, HBKU, Doha, Qatar

V. K. Narayanan Department of Management, LeBow College of Business, Drexel University, Philadelphia, PA, USA

Franco Maria Nardini ISTI-CNR, Pisa, Italy

Daniel B. Neill Event and Pattern Detection Laboratory, H. J. Heinz III College, Carnegie Mellon University, Pittsburgh, PA, USA

Maximilian Nickel Department of Computer Science, Ludwig Maximilian University of Munich, München, Germany

Thomas Niebler Data Mining and Information Retrieval Group, University of Wuerzburg, Wuerzburg, Germany

Malvina Nissim University of Groningen, Groningen, The Netherlands

Nicole Novielli Department of Computer Science, University of Bari Aldo Moro, Bari, Italy 
Eirini Ntoutsi Department of Electrical Engineering and Computer Science, Leibniz University Hannover, Hannover, Germany

Ludwig Maximilian Univ. Munich, Munich, Germany

Darko Obradović Knowledge Management, German Research Center for Artificial Intelligence (DFKI), Kaiserslautern, Germany

Jae C. Oh Department of Electrical Engineering and Computer Science, Syracuse University, Syracuse, NY, USA

Kieron O'Hara Web and Internet Science Group, Electronics and Computer Science, University of Southampton, Southampton, UK

Victor Oliveira Department of Management Science and Statistics, The University of Texas at San Antonio, San Antonio, TX, USA

Dev Oliver Department of Computer Science, University of Minnesota, Minneapolis, MN, USA

Sirinya On-At Toulouse Institute of Computer Science Research, University of Toulouse, CNRS, INPT, UPS, UT1, UT2J, Toulouse, France

Systèmes d'Informations Généralisés, Institut de Recherche en Informatique de Toulouse (IRIT), Toulouse, France

Makoto Onizuka Graduate School of Information Science and Technology, Osaka University, Osaka, Japan

Anthony J. Onwuegbuzie Department of Educational Leadership, Sam Houston State University, Huntsville, TX, USA

Faculty of Education, University of Johannesburg, Auckland Park, South Africa

Alen Orbanić Faculty of Mathematics and Physics, University of Ljubljana, Ljubljana, Slovenia

Physics and Mechanics, Institute of Mathematics, Ljubljana, Slovenia

Andrej Marušič Institute, University of Primorska, Koper, Slovenia

Abelium d.o.o., Research and Development, Ljubljana, Slovenia

G. K. Orman Engineering Faculty, Computer Engineering Department, Galatasaray University, Istanbul, Turkey

Lukasz Ostrowski Warsaw University, Warsaw, Poland

Mark Carroll Pachucki Department of Sociology, Computational Social Science Institute, University of Massachusetts, Amherst, Amherst, MA, USA

Darren Palmer Criminology, School of Humanities and Social Sciences, Deakin University, Geelong, VIC, Australia

Arun Pandey Indian Institute of Technology, Patna, Bihar, India

Symeon Papadopoulos Centre for Research and Technology Hellas, Information Technologies Institute, Thessaloniki, Greece 
Georgios Papageorgiou Department of Information and Communication Systems Engineering, University of the Aegean, Karlovassi, Samos, Greece

Evangelos E. Papalexakis Computer Science and Engineering Department, UC Riverside, Riverside, CA, USA

School of Computer Science, Carnegie Mellon University, Pittsburgh, PA, USA

Abigail Paradise Department of Software and Information Systems Engineering, Ben-Gurion University of the Negev, Beer-Sheva, Israel

Jan Paredaens Department of Math and Computer Science, University of Antwerp, Antwerp, Belgium

Han Woo Park Department of Media and Information, Interdisciplinary Program of Digital Convergence Business, YeungNam University, GyeongSan, Korea

Davide Maria Parrilli time.lex law firm, Brussels, Belgium

Viviana Patti Department of Computer Science, University of Turin, Turin, Italy

Elena Pavan Institute of Humanities and Social Sciences, Scuola Normale Superiore, Florence, Italy

Dino Pedreschi KDDLab, University of Pisa, Pisa, Italy

Konstantinos Pelechrinis School of Information Sciences, University of Pittsburgh, Pittsburgh, PA, USA

André Péninou Toulouse Institute of Computer Science Research, University of Toulouse, CNRS, INPT, UPS, UT1, UT2J, Toulouse, France

Systèmes d'Informations Généralisés, Institut de Recherche en Informatique de Toulouse (IRIT), Toulouse, France

Raffaele Perego ISTI-CNR, Pisa, Italy

Charles Perez PSB Paris School of Business, Chair Digital Data Design, Paris, France

Alexander Perrone Applied Physics Laboratory, Johns Hopkins University, Laurel, MD, USA

Jérôme Picault Alcatel-Lucent Bell Labs, Nozay, France

Bruno Pinaud CNRS UMR 5800 LaBRI, University of Bordeaux, Talence, France

Mahendra Piraveenan Complex Systems Research Group, Project Management Program, Faculty of Engineering and Information Technologies, The University of Sydney, Sydney, NSW, Australia

A. Pluhár Institute of Informatics, University of Szeged, Szeged, Hungary 
Marco Polignano Department of Computer Science, University of Bari Aldo Moro, Bari, Italy

Axel Polleres Siemens AG Österreich, Vienna, Austria

James Ponder School of Communication Studies, Kent State University, Kent, OH, USA

Soujanya Poria School of Computer Science and Engineering, Nanyang Technological University, Singapore, Singapore

Andrea Pugliese DIMES Department, University of Calabria, Rende, Italy

Hemant Purohit Ohio Center of Excellence in Knowledge-Enabled Computing (Kno.e.sis), Wright State University, Dayton, OH, USA

George Mason University, Fairfax, VA, USA

Rami Puzis University of Maryland Institute for Advanced Computer Studies (UMIACS), College Park, MD, USA

Department of Software and Information Systems Engineering, Ben-Gurion University of the Negev, Beer-Sheva, Israel

Yuyang Qiu College of Statistics and Mathematics, Zhejiang Gongshang University, Hangzhou, China

Walter Quattrociocchi London Institute of Mathematical Sciences, London, Mayfair, UK

Natwork Department, IMT Alti Studi Lucca, Lucca, Italy

Jörg Raab Department of Organization Studies, Tilburg University, Tilburg, The Netherlands

Elie Raad Faculty of Business, Memorial University of Newfoundland, St. John's, NL, Canada

Reihaneh Rabbany Computing Science Department, University of Alberta, Edmonton, AB, Canada

Huzefa Rangwala Department of Computer Science, George Mason University, Fairfax, VA, USA

Sylvie Ratté École de technologie supérieure, Montreál, QC, Canada

Miriam Redi Social Dynamics Team, Nokia Bell Labs, Cambridge, UK

Benjamin Renoust Digital Content and Media Sciences Research, National Institute of Informatics and CNRS UMI 3527 JFLI, Tokyo, Japan

Chiara Renso ISTI Institute of National Research Council, Pisa, Italy

Pedro Ribeiro CRACS and INESC-TEC, Faculdade de Ciencias, Universidade do Porto, Porto, Portugal 
Francesco Ricci Faculty of Computer Science, Free University of BozenBolzano, Bozen-Bolzano, Italy

Mirela Riveni Distributed Systems Group, Institute for Information Systems, TU Wien, Vienna, Austria

Aleksei Romanov Faculty of Information Technology, University of Jyväskylä, Jyväskylä, Finland

Giulio Rossetti KDD Lab, University of Pisa, Pisa, Italy

Luca Rossi University of Copenhagen, København, Denmark

Maytham Safar College of Engineering and Petroleum, Kuwait University, Kuwait City, Kuwait

Stanisław Saganowski Department of Computational Intelligence, Wrocław University of Science and Technology, Wrocław, Poland

Tanwistha Saha Technology Manufacturing Group (TMG), Intel Corporation, Hillsboro, OR, USA

Alan Said School of Informatics, University of Skövde, Skövde, Sweden

Arnaud Sallaberry LIRMM - Université Paul Valéry, Montpellier, France

J. Alfredo Sánchez French-Mexican Laboratory of Informatics and Automatic Control (LAFMIA, UMI CNRS 3175), Lyon, France

Peter Sanders Institute of Theoretical Informatics, Karlsruhe Institute of Technology (KIT), Karlsruhe, Germany

Mauro San Martín Department of Mathematics, Universidad de La Serena, La Serena, Chile

Giuseppe Sansonetti Department of Engineering, Roma Tre University, Rome, Italy

Chandan Sarkar Department of Telecommunication, Information Studies and Media, Michigan State University, East Lansing, MI, USA

Soumya Sarkar Indian Institute of Technology, Patna, Bihar, India

Idrissa Sarr Department of Computer Science and Mathematics, Université Cheikh Anta Diop, Dakar-Fann, Sénégal

Nishanth Sastry Department of Informatics, King's College London, London, UK

Aarti Sathyanarayana Department of Computer Science and Engineering, University of Minnesota, Minneapolis, MN, USA

Ali Sayfy Department of Mathematics and Statistics, American University of Sharjah, Sharjah, UAE

Ognjen Scekic Distributed Systems Group, Institute for Information Systems, TU Wien, Vienna, Austria 
Daniel Schall Siemens Corporate Technology, Vienna, Austria

Maximilian Schich School of Arts, Technology, and Emerging Communication, The University of Texas at Dallas, Richardson, TX, USA

Christoph Schlieder University of Bamberg, Faculty for Information Systems and Applied Computer Sciences, Chair of Computing in the Cultural Sciences, Bamberg, Germany

Thomas Schøtt Department of Entrepreneurship and Relationship Management, University of Southern Denmark, Kolding, Denmark

Christian Schulz Institute of Theoretical Informatics, Karlsruhe Institute of Technology (KIT), Karlsruhe, Germany

Frank Schweitzer Chair of Systems Design, ETH Zurich, Zurich, Switzerland

Fabrizio Sebastiani Istituto di Scienza e Tecnologie dell'Informazione, Consiglio Nazionale delle Ricerche, Pisa, Italy

Florence Sèdes Toulouse Institute of Computer Science Research, University of Toulouse, CNRS, INPT, UPS, UT1, UT2J, Toulouse, France

Systèmes d'Informations Généralisés, Institut de Recherche en Informatique de Toulouse (IRIT), Toulouse, France

Alexander Semenov Faculty of Information Technology, University of Jyväskylä, Jyväskylä, Finland

Kunal Sengupta Data Semantics Laboratory, Department of Computer Science and Engineering, Wright State University, Dayton, OH, USA

Sebastian Sevignani Unified Theory of Information Research Group (UTI), Vienna, Austria

Institute of Sociology, Friedrich-Schiller-University Jena, Jena, Germany

Andres Sevtsuk Graduate School of Design, City Form Lab, Harvard University, Cambridge, MA, USA

City Form Lab, Singapore University of Technology and Design, Singapore, Singapore

Asaf Shabtai Department of Software and Information Systems Engineering, Ben-Gurion University of the Negev, Beer-Sheva, Israel

Shashi Shekhar Department of Computer Science, University of Minnesota, Minneapolis, MN, USA

Amit Sheth Ohio Center of Excellence in Knowledge-Enabled Computing (Kno.e.sis), Wright State University, Dayton, OH, USA

Hiroaki Shiokawa Center for Computational Sciences, University of Tsukuba, Ibaraki, Japan

Maryam Shoaran Department of Mechatronics, School of EngineeringEmerging Technologies, University of Tabriz, Tabriz, Iran 
Pavel Shvaiko Informatica Trentina SpA, Trento, Italy

Fernando Silva CRACS and INESC-TEC, Faculdade de Ciencias, Universidade do Porto, Porto, Portugal

Philipp Singer Knowledge Technologies Institute, Graz University of Technology, Graz, Austria

Lisa Singh Department of Computer Science, Georgetown University, Washington, DC, USA

Sumit Singh Software Engineering Department, Directi, Gurgaon, India

Florian Skopik Digital Safety and Security Department, AIT Austrian Institute of Technology, Vienna, Austria

Marc A. Smith Social Media Research Foundation, Redwood City, Belmont, CA, USA

Gary Alan Smith Ohio Center of Excellence in Knowledge-Enabled Computing (Kno.e.sis), Wright State University, Dayton, OH, USA

Robert R. Snapp Department of Computer Science, University of Vermont, Burlington, VT, USA

Tom A. B. Snijders Department of Sociology, University of Groningen, Groningen, The Netherlands

Department of Statistics and Nuffield College, University of Oxford, Oxford, UK

Sriram Somanchi Event and Pattern Detection Laboratory, H. J. Heinz III College, Carnegie Mellon University, Pittsburgh, PA, USA

Zhao Song School of Computing Science, Simon Fraser University, Burnaby, BC, Canada

Skyler Speakman Event and Pattern Detection Laboratory, H. J. Heinz III College, Carnegie Mellon University, Pittsburgh, PA, USA

Sargur N. Srihari Department of Computer Science and Engineering, University at Buffalo, The State University of New York, Buffalo, NY, USA

Venkatesh Srinivasan Department of Computer Science, University of Victoria, Victoria, BC, Canada

Gautam Srivastava Department of Math and Computer Science, Brandon University, Brandon, MB, Canada

Jaideep Srivastava Department of Computer Science and Engineering, University of Minnesota, Minneapolis, MN, USA

Steffen Staab Institute for Web Science and Technologies, Universität Koblenz-Landau, Koblenz, Germany 
Johann Stan Univ. Lyon, UJM-Saint-Etienne, CNRS, Laboratoire Hubert Curien UMR 5516, Saint Etienne, France

ISCOD - Institut Henri Fayol, Ecole Nationale Supérieure des Mines, Université de Lyon, UJM-Saint-Étienne, CNRS, Saint-Étienne, France

Milan Stankovic Université Paris-Sorbonne, Paris, France

Peter Steenkiste School of Computer Science, Carnegie Mellon University, Pittsburgh, PA, USA

Department of Electrical and Computer Engineering, Carnegie Mellon University, Pittsburgh, PA, USA

Krzysztof Stefaniak Institute of Computing Science, Poznan University of Technology, Poznań, Poland

Kostas Stefanidis School of Information Sciences, University of Tampere, Tampere, Finland

Mary Stegmaier Truman School of Public Affairs, University of Missouri, Columbia, MO, USA

Benno Stein Bauhaus-Universität Weimar, Weimar, Germany

Stefan Stieglitz Department of Computer Science and Applied Cognitive Science, Professional Communication in Electronic Media/Social Media, University of Duisburg-Essen, Duisburg, Germany

Frans N. Stokman Department of Sociology, Interuniversity Center for Social Science Theory and Methodology, University of Groningen, Groningen, The Netherlands

Markus Strohmaier Computational Social Science Group, GESIS - Leibniz Institute for the Social Sciences, Cologne, Germany

Department of Computer Science, University of Koblenz-Landau, Koblenz, Germany

V. S. Subrahmanian Computer Science Department, University of Maryland, College Park, MD, USA

Eric A. Suess Department of Statistics and Biostatistics, California State University, East Bay, Hayward, CA, USA

Daniel Sui Department of Geography, The Ohio State University, Columbus, $\mathrm{OH}$, USA

Yizhou Sun College of Computer and Information Science, Northeastern University, Boston, MA, USA

Didi Surian School of Information Technologies, University of Sydney, Sydney, NSW, Australia

Centre for Health Informatics, Australian Institute of Health Innovation, Macquarie University, Sydney, NSW, Australia 
Stefan Tai Information Systems Engineering Research Group, Fakultät IV Elektrotechnik und Informatik, TU Berlin, Berlin, Germany

Mansoreh Takaffoli Computing Science Department, University of Alberta, Edmonton, AB, Canada

Jie Tang Department of Computer Science and Technology, Tsinghua University, Beijing, China

Haicheng Tao College of Computer Science and Engineering, Nanjing University of Science and Technology, Nanjing, China

Tzouramanis Theodoros Department of Information and Communication Systems Engineering, University of the Aegean, Karlovassi, Samos, Greece

Marie Thilliez LAMIH UMR CNRS/UVHC 8201, University Lille North of France, Valenciennes, France

Alex Thomo Department of Computer Science, University of Victoria, Victoria, BC, Canada

Christophe Thovex French-Mexican Laboratory of Informatics and Automatic Control (LAFMIA, UMI CNRS 3175), Lyon, France

Rupa Tiwari Department of Computer Science, University of Minnesota, Minneapolis, MN, USA

Marko Tkalčič Faculty of Computer Science, Free University of BozenBolzano, Bozen-Bolzano, Italy

Emanuela Todeva Research Centre for Business Clusters, Networks, and Economic Development, London, UK

Mario Vincenzo Tomasello Chair of Systems Design, ETH Zurich, Zurich, Switzerland

Ernst \& Young, Fraud Investigation and Dispute Services, Zurich, Switzerland

Paolo Tomeo SisInf Lab, Polytechnic University of Bari, Bari, Italy

Hanghang Tong CUNY City College, New York, NY, USA

René Torenvlied Department of Public Administration, University of Twente, Enschede, The Netherlands

Carmen Torrano ElevenPaths, Malaga, Spain

Radhia Toujani Department of Computer Science, Bestmod Laboratory, ISG Tunis Université de Tunis, Tunis, Tunisia

Masashi Toyoda Institute of Industrial Science, University of Tokyo, Tokyo, Meguro $\mathrm{Ku}$, Japan

Christoph Trattner Knowledge Management Institute and Institute for Information Systems and Computer Media, Graz University of Technology, Graz, Austria 
Volker Tresp Corporate Technology, Siemens AG, München, Germany

Department of Computer Science, Ludwig Maximilian University of Munich, München, Germany

Francky Trichet Laboratory of Computer Sciences (LINA, UMR CNRS 6241), University of Nantes, Nantes, France

A. Alexandre Trindade Department of Mathematics and Statistics, Texas Tech University, Lubbock, TX, USA

Peter Tröger Computer Science, Chemnitz University of Technology, Chemnitz, Germany

Bruce E. Trumbo Department of Statistics and Biostatistics, California State University, East Bay, Hayward, CA, USA

Hong-Linh Truong Distributed Systems Group, Institute for Information Systems, TU Wien, Vienna, Austria

Piotr Turek Faculty of Informatics, Polish-Japanese Institute of Information Technology, Warsaw, Poland

Theodoros Tzouramanis Department of Information and Communication Systems Engineering, University of the Aegean, Karlovassi, Samos, Greece

Shahadat Uddin Complex Systems Research Group Faculty of Engineering and IT, The University of Sydney, Sydney, NSW, Australia

Fatemeh Vahedian College of Computing and Digital Media, DePaul University, Chicago, IL, USA

Athena Vakali Department of Informatics, Aristotle University of Thessaloniki, Thessaloniki, Greece

Jason Vallet CNRS UMR 5800 LaBRI, University of Bordeaux, Talence, France

Wil M. P. van der Aalst Department of Mathematics and Computer Science, Eindhoven University of Technology, Eindhoven, The Netherlands

Dirk Pieter van Donk Department Operations, Faculty of Economics and Business, University of Groningen, Groningen, The Netherlands

Karl M. van Meter Centre Maurice Halbwachs, Social Sciences Department, Ecole Normale Supérieure, Paris, France

Kees van Veen Faculty of Economics and Business, University of Groningen, Groningen, The Netherlands

Kush R. Varshney Mathematical Sciences Department, IBM Thomas J. Watson Research Center, Yorktown Heights, NY, USA

Mahadevan Vasudevan EMC Corp, Hopkinton, MA, USA

Jari Veijalainen Faculty of Information Technology, University of Jyväskylä, Jyväskylä, Finland 
Yannis Velegrakis Department of Information Engineering and Computer Science, University of Trento, Trento, Italy

Ted Vickey Digital Enterprise Research Institute, National University of Ireland at Galway, Galway, Ireland

Gerard Vidal Enigmedia, Menlo Park, CA, USA

Jessica Vitak College of Information Studies, University of Maryland, College Park, MD, USA

Chris Volinsky Statistics Research Department, AT\&T Labs-Research, Florham Park, NJ, USA

Beate Volker Department of Sociology, University of Amsterdam, Amsterdam, The Netherlands

Eleni Vourou Department of Information and Communication Systems Engineering, University of the Aegean, Karlovassi, Samos, Greece

Monica Wachowicz University of New Brunswick, People in Motion Lab, Fredericton, Canada

Dorothea Wagner Institute of Theoretical Informatics, Karlsruhe Institute of Technology (KIT), Karlsruhe, Germany

William A. Wallace Department of Industrial and Systems Engineering, Rensselaer Polytechnic Institute, Troy, NY, USA

Chi Wang Microsoft Research, Redmond, WA, USA

Dashun Wang Kellogg School of Management, Northwestern University, Evanston, IL, USA

Northwestern Institute on Complex Systems (NICO), Northwestern University, Evanston, IL, USA

Fei Wang Healthcare Analytics Research Group, IBM T. J. Watson Research Center, Yorktown Heights, NY, USA

Jun Wang School of Computer Science and Software Engineering, East China Normal University, Shanghai, China

Wenbo Wang Ohio Center of Excellence in Knowledge-Enabled Computing (Kno.e.sis), Wright State University, Dayton, OH, USA

GoDaddy, Inc., San Francisco, CA, USA

Youquan Wang Jiangsu Provincial Key Laboratory of E-Business, Nanjing University of Finance and Economics, Nanjing, China

Zhefeng Wang School of Computer Science and Technology, University of Science and Technology of China, Anhui, China

Ian Warren Criminology, School of Humanities and Social Sciences, Deakin University, Geelong, VIC, Australia 
Jarosław Wąs Faculty of Electrical Engineering, Automatics, Computer Science and Biomedical Engineering, Department of Applied Computer Science, AGH University of Science and Technology, Kraków, Poland

Ingo Weber Data61, CSIRO, Sydney, Australia

Jens Weber Department of Computer Science, University of Victoria, Victoria, BC, Canada

Zhen Wen Alibaba Cloud, Hangzhou, Zhejiang, China

Adam Wierzbicki Faculty of Informatics, Polish-Japanese Institute of Information Technology, Warsaw, Poland

Rolf T. Wigand Departments of Information Science and Management, University of Arkansas Little Rock (UALR), Little Rock, AR, USA

Lynne J. Williams BC Children's Hospital MRI Research Facility, Vancouver, BC, Canada

Stephen Wilson Lockstep Consulting, Sydney, NSW, Australia

Australian Cyber Security Centre, University of New South Wales, Canberra, NSW, Australia

Constellation Research, San Francisco, CA, USA

Rafael Wittek Theoretical Sociology - Department of Sociology, University of Groningen, Groningen, The Netherlands

Donghee Yvette Wohn Department of Telecommunication, Information Studies and Media, Michigan State University, East Lansing, MI, USA

Liang Wu Data Mining and Machine Learning Lab, School of Computing, Informatics, and Decision Systems Engineering, Arizona State University, Tempe, AZ, USA

Zhiang Wu Jiangsu Provincial Key Laboratory of E-Business, Nanjing University of Finance and Economics, Nanjing, China

Yanghua Xiao School of Computer Science, Fudan University, Shanghai, China

Hui Xiong Department of Management Science and Information Systems, Rutgers the State University of New Jersey, Newark, NJ, USA

Bo Xu School of Computer Science, Fudan University, Shanghai, China

Feng Xu State Key Laboratory for Novel Software Technology, Nanjing University, Nanjing, Jiangsu, China

Guandong Xu Advanced Analytics Institute, University of Technology, Sydney, Broadway, NSW, Australia

Jennifer J. Xu Department of Computer Information Systems, Bentley University, Waltham, MA, USA 
Ronghua Xu College of Economics and Management, Zhejiang University of Technology, Hangzhou, Zhejiang Province, China

Zengwang Xu Department of Geography, University of Wisconsin-Milwaukee, Milwaukee, WI, USA

Elina Yaakobovich Department of Information Systems Engineering, BenGurion University of the Negev, Beer-Sheva, Israel

Erjia Yan College of Computing and Informatics, Drexel University, Philadelphia, PA, USA

Deqing Yang School of Data Science, Fudan University, Shanghai, China

Li Yang Department of Computer Science and Engineering, The University of Tennessee-Chattanooga, Chattanooga, TN, USA

Tianbao Yang Machine Learning Lab, GE Global Research, San Ramon, CA, USA

Yang Yang Northwestern Institute on Complex Systems (NICO), Northwestern University, Evanston, IL, USA

Kellogg School of Management, Northwestern University, Evanston, IL, USA

Yuan Yao State Key Laboratory for Novel Software Technology, Nanjing University, Nanjing, Jiangsu, China

Mao Ye Pinterest, Inc., San Francisco, CA, USA

Aylin Yener Department of Electrical Engineering, The Pennsylvania State University, State College, PA, USA

Guojian Yin Department of Mathematics, The Chinese University of Hong Kong, Shatin, NT Hong Kong SAR, China

Hongzhi Yin School of Information Technology and Electrical Engineering, The University of Queensland, Brisbane, QLD, Australia

Man Lung Yiu The Department of Computing, The Hong Kong Polytechnic University, Hung Hom, Hong Kong

Philip S. Yu Department of Computer Science, University of Illinois at Chicago, Chicago, IL, USA

Quan Yuan Department of Computer Science, University of Illinois at Urbana-Champaign, Champaign, IL, USA

Osmar R. Zaïane Computing Science Department, University of Alberta, Edmonton, AB, Canada

Faraz Zaidi MetricAid Inc., North Bay, Canada

Karachi Institute of Economics and Technology (KIET), Karachi, Pakistan

Sam Zeini Department of Computer Science and Applied Cognitive Science, University of Duisburg-Essen, Duisburg, Germany

Hongyi Zhang Microsoft, Vancouver, BC, Canada 
Jiawei Zhang Department of Computer Science, Florida State University, Tallahassee, FL, USA

Lei Zhang LinkedIn, Sunnyvale, CA, USA

Lu Zhang Jiangsu Provincial Key Laboratory of E-Business, Nanjing University of Finance and Economics, Nanjing, China

Qingpeng Zhang Department of Systems Engineering and Engineering Management, City University of Hong Kong, Hong Kong, SAR, China

Shenzhen Research Institute of City University of Hong Kong, Shenzhen, China

Qingqing Zhang School of Computer Science and Technology, University of Science and Technology of China, Hefei, China

Xiao-Dong Zhang School of Mathematical Science, Ministry of Education Key Laboratory of Scientific and Engineering Computing, Shanghai Jiao Tong University, Shanghai, China

Bo Zhao Department of Geography, The Ohio State University, Columbus, $\mathrm{OH}$, USA

Kaiqi Zhao School of Computer Engineering, Nanyang Technological University, Singapore, Singapore

Peilin Zhao Ant Financial, Hangzhou, China

Shenglin Zhao The Chinese University of Hong Kong, Hong Kong, China

Bin Zhou Department of Information Systems, University of Maryland, Baltimore County, Baltimore, MD, USA

Jiaqi Zhou Department of Systems Engineering and Engineering Management, City University of Hong Kong, Hong Kong, SAR, China

Xiaofang Zhou School of Information Technology and Electrical Engineering, The University of Queensland, Brisbane, QLD, Australia

Shenghuo Zhu NEC Laboratories America, Inc., Cupertino, CA, USA

Yada Zhu IBM Research, Yorktown Heights, NY, USA

Katharina Anna Zweig Department of Computer Science, Complex Network Analysis and Graph Theory, University of Kaiserslautern, Kaiserslautern, Germany

Anna Zygmunt Department of Computer Science, AGH University of Science and Technology, Kraków, Poland 